\title{
CHAIN TRANSITIVE HOMEOMORPHISMS ON A SPACE: ALL OR NONE
}

\author{
ETHAN AKIN AND JUHO RAUTIO
}

\begin{abstract}
We consider which spaces can be realized as the omega limit set of the discrete time dynamical system. This is equivalent to asking which spaces admit a chain transitive homeomorphism and which do not. This leads us to ask for spaces where all homeomorphisms are chain transitive.
\end{abstract}

\section{Introduction}

We will use the term space to mean a nonempty, compact metrizable space unless otherwise mentioned. When a metric is required we, assume that one is chosen and fixed. Where a metric is used in a construction below, the result is independent of the choice of metric. Broadly, our metric space ideas and constructions are really uniform space concepts and a compact space has a unique uniformity.

The dynamical systems we will consider are pairs $(X, f)$, where $f$ : $X \rightarrow X$ is a continuous map on a space $X$. If $x \in X$, then the sequence $\left\{f(x), f^{2}(x), \ldots\right\}$ is the positive $f$-orbit sequence of $x$ and $\omega f(x)$ is the set of limit points of the sequence.

In a series of papers, [1], [2] and [3], Agronsky and his collaborators initiated study of the omega set problem. Call the pair $(X, f)$ an omega system when it can be embedded as a subsystem of some $(Y, g)$ so that $X=\omega g(y)$ for some $y \in Y$. The authors call the system orbit enclosing when one can choose $(Y, g)=(X, f)$, i.e. there exists $x \in X$ such that $\omega f(x)=X$. A space $X$ is an omega set when there exists a continuous map $f$ on $X$ so that $(X, f)$ is an omega system.

In current parlance, the system $(X, f)$ is an orbit enclosing omega system exactly when it is topologically transitive. In general, the system $(X, f)$ is an omega system exactly when it is chain transitive.

Date: June, 2015.

Key words and phrases. omega set, chain transitive homeomorphism, rigid space, Slovak space.

2010 Mathematical Subject Classification 37B20, 37B25 . 
Given $\epsilon \geq 0$, a finite or infinite sequence $\left\{x_{n} \in X\right\}$ with at least two terms is an $\epsilon$-chain for $(X, f)$ if $d\left(f\left(x_{k}\right), x_{k+1}\right) \leq \epsilon$ for all terms $x_{k}$ of the sequence (except the last one). The system $(X, f)$ is called chain transitive when every pair of points of $X$ can be connected by some finite $\epsilon$-chain for every positive $\epsilon$. A subset $A \subset X$ is called a chain transitive subset when it is closed and $f$-invariant and the subsystem $(A, f)$ is chain transitive.

It is well known that any omega limit set is a chain transitive subset, e.g. [4] Proposition 4.14. On the other hand, as observed by Takens, it is an easy exercise to show that if $(X, f)$ is chain transitive, then then it can be embedded in a larger system in which it is an omega limit set, 4 Exercise 4.29. We will review the proofs in Section 3. The construction uses a subset $Y$ of $X \times[0,1]$. In particular, if $X \subset[0,1]^{n}$ then $Y \subset[0,1]^{n+1}$. Applying the Tietze Extension Theorem in each coordinate, we can extend $g$ to a continuous map on all of $[0,1]^{n+1}$ and so obtain $X$ as the omega limit set of a system on $[0,1]^{n+1}$. Note, however, that even if $g$ is a homeomorphism, we might not be able to extend it to a homeomorphism on $[0,1]^{n+1}$.

This equivalence is really a classical result due to Dowker and Friedlander [12] for homeomorphisms and to Sarkovskii [20] in general. They, however, use a different but equivalent condition instead of chain transitivity. They say that $(X, f)$ is $f$-connected if, for any proper, nonempty, closed subset $U \subset X$, the intersection $f(U) \cap \overline{X \backslash U} \neq \emptyset$. They show that $(X, f)$ is an omega system iff it is $f$-connected.

To clarify the relationship between chain transitivity and $f$-connectedness, we recall the concept of an attractor, as described by Conley in [9] and with detailed exposition in [4]. Call a closed set $U \subset X$ an inward set for $f$ if $f(U)$ is contained in the interior $U^{\circ}$ or, equivalently, $f(U) \cap \overline{X \backslash U}=\emptyset$. Thus, $(X, f)$ admits a proper, nonempty, inward subset iff it is not $f$-connected. If $U$ is an inward set, then $A=\bigcap_{n=0}^{\infty} f^{n}(U)$ is called the associated attractor. For a number of equivalent descriptions of an attractor see [4] Theorem 3.3. Theorem 4.12 of [4] says that $(X, f)$ is chain transitive iff $X$ is the only nonempty attractor and iff $X$ is the only nonempty inward set. It follows that chain transitivity and $f$-connectedness are equivalent concepts.

The label "attractor" has been used for other ideas. Some authors refer to all omega limit sets as attractors. An attractor as defined above need not be chain transitive, and so there are attractors which are not omega systems. A more reasonable definition is that a set $A$ is an attractor for $f$ when it is $f$-invariant and, for every $x$ in some neighborhood of $A$, we have $\omega f(x) \subset A$. This condition is necessary 
in order that $A$ be an attractor à la Conley, but it is not sufficient. If $X$ is the one-point compactification of $\mathbb{Z}$ and $f$ is the extension to $X$ of translation by 1 on $\mathbb{Z}$, then $(X, f)$ is chain transitive. On the other hand, the point at infinity is the omega limit set of every point. By Theorem 3.6(a) of [4], an $f$-invariant subset $A$ is an attractor iff $\{x: \omega f(x) \subset A\}$ is a neighborhood of $A$ and, in addition, $A$ is stable, i.e. for every $\epsilon>0$ there exists $\delta>0$ such that if $x$ is $\delta$-close to $A$, then the forward orbit of $x$ remains $\epsilon$-close to $A$.

The identity map $1_{X}$ on $X$ is chain transitive iff $X$ is connected. Thus, a connected space admits a chain transitive homeomorphism and so is an omega space.

Recall that a Peano space is a compact, connected, locally connected space or, equivalently, a continuous image of the unit interval. The major result of [3] is that if $X$ has finitely many components and each is a nontrivial, finite-dimensional Peano space, then $X$ is an orbit enclosing omega space. That is, it admits a topologically transitive map.

Our purpose here is to consider the homeomorphism version of the omega set problem. That is, we ask when a space $X$ admits a homeomorphism $f$ such that $(X, f)$ is an omega system or, equivalently, such that $f$ is a chain transitive map.

To illustrate the difference between the original problem and the homeomorphism version, consider the tent map on $[0,1]$ defined by

$$
T(t)= \begin{cases}2 t & \text { for } 0 \leq t \leq \frac{1}{2} \\ 1-2 t & \text { for } \frac{1}{2} \leq t \leq 1\end{cases}
$$

which is well known to be topologically transitive. Now let $X_{0}=$ $[0,1] \times\{0,1\}$, and define $f_{0}$ on $X_{0}$ by

$$
f_{0}(t, 0)=(t, 1) \quad \text { and } \quad f_{0}(t, 1)=(T(t), 0),
$$

so that $f_{0}^{2}=T \times 1_{\{0,1\}}$.

Let $(X, f)$ be the quotient system obtained by identifying the points $(0,1)=(1,1)$ in $X$. Thus, $X$ is the disjoint union of a circle and an interval. It easily follows that $X$ does not admit a chain transitive homeomorphism. On the other hand, $f$ is a topologically transitive map.

Let $\left(X_{1}, f_{1}\right)$ be the quotient system obtained by identifying the points $(0,0)=(0,1)=(1,1)$ in $X_{1}$. Thus, $X_{1}$ consists of a circle and an interval joined at a point. As $X_{1}$ is connected, the identity is a chain transitive homeomorphism. Since the points of the interval other than $(1,0)$ separate the space and the points of the circle other than the intersection point with the interval do not, it easily follows that $X_{1}$ 
does not admit a topologically transitive homeomorphism. On the other hand, $f_{1}$ is a topologically transitive map.

If $X$ contains a proper, clopen, nonempty, $f$-invariant set $A$, then we say that $X$ is $f$-decomposable. In this case $(X, f)$ is not chain transitive, for if $\epsilon$ is smaller than the distance from $A$ to its complement, then any $\epsilon$-chain which begins in $A$ remains in $A$. With $H(X)$ the group of homeomorphisms on $X$, we say that $X$ is $H(X)$-decomposable if there is a proper, clopen, nonempty subset $A$ of $X$ such that $A$ is invariant for every homeomorphism on $X$. Clearly, if $X$ is $H(X)$-decomposable, then it admits no chain transitive homeomorphism. For example, let $\operatorname{Iso}(X)$ denote the (possibly empty) set of isolated points of $X$. If the closure $\overline{\mathrm{Iso}(X)}$ is a proper, clopen, nonempty subset of $X$, then $X$ is $H(X)$ decomposable and so admits no chain transitive homeomorphism. In the zero-dimensional case, this is the only obstruction. We prove a slightly more general result in Section 3 .

If $X$ is not $f$-decomposable (or not $H(X)$-decomposable), we will call it $f$-indecomposable (resp. $H(X)$-indecomposable).

Theorem 1.1. If $X$ is a space such that $\overline{\operatorname{Iso}(X)}$ is not a proper clopen subset of $X$ and such that the open set $X \backslash \overline{\mathrm{Iso}(X)}$ is empty or zerodimensional, then $X$ admits a chain transitive homeomorphism.

Corollary 1.2. If the isolated points are dense in $X$, then $X$ admits a chain transitive homeomorphism.

Thus, the problems which remain come from the nontrivial components. A clopen component is called an isolated component. Clearly, if the closure of the isolated components is a proper, clopen, nonempty subset of $X$, then $X$ is $H(X)$-decomposable.

If $y$ is a set of connected spaces, let $C y$ denote the closure of the union of those components of $X$ which are homeomorphic to some element of $y$. If $C_{y}$ is a proper, clopen, nonempty subset, then $X$ is $H(X)$ decomposable. For example, if $X$ has finitely many components, then either they are all homeomorphic, in which case $X$ admits a periodic chain transitive map, or $X$ is $H(X)$-decomposable. Contrast this with the map result described above.

We say that $X$ satisfies the diameter condition on isolated components if for every $\epsilon>0$ there are only finitely many isolated components with diameter greater than $\epsilon$.

Theorem 1.3. If $X$ satisfies the diameter condition and the union of the isolated components is dense in $X$, then either $X$ is $H(X)$ decomposable or else $X$ admits a chain transitive homeomorphism. 
The rest of Section 3 consists of counterexamples to reasonable conjectures. We construct

- A space $X$ that is $H(X)$-decomposable, with all components homeomorphic, and no isolated components.

- A space $X$ that is $H(X)$-indecomposable but $f$-decomposable for every $f \in H(X)$. The space can be chosen with the isolated components all homeomorphic and with a dense union.

- A space $X$ that is $f$-indecomposable for some $f \in H(X)$ but admits no chain transitive homeomorphism. The space can be chosen with the isolated components all homeomorphic and with a dense union.

These examples rule out the obvious extension of the above corollary. The isolated components can be dense and all homeomorphic to one another, but nonetheless the space admits no chain transitive homeomorphism.

Having considered when there are no chain transitive homeomorphisms, we consider in Section 4 the question: When is every homeomorphism on a space $X$ chain transitive? For such a space $X$, the identity map $1_{X}$ is chain transitive, and so $X$ must be connected.

In [11, rigid spaces were defined and Peano space examples were constructed. A space $X$ is rigid if $1_{X}$ is the only homeomorphism on $X$, i.e. the homeomorphism group $H(X)$ is trivial. For a connected rigid space, it is trivially true that all homeomorphisms are chain transitive.

Using rigid spaces one can construct more interesting examples. Following [10], we can begin with a finitely generated group $G$ and use rigid spaces instead of intervals as edges in the Cayley graph. If $X$ is the one-point compactification of this fattened Cayley graph, then $H(X)$ is isomorphic to $G$ and every homeomorphism is chain transitive. We obtain examples with non-discrete homeomorphism group and even with the path components nontrivial.

In these cases, the homeomorphism group does not act in a topologically transitive manner on the space. Distinct points in each rigid piece are homeomorphically distinct. It is possible to obtain examples with all homeomorphisms chain transitive and with the homeomorphism group acting in a topologically transitive manner. These are built using the recent, beautiful construction in [13] of Slovak spaces. A Slovak space $X$ has $H(X)$ isomorphic to $\mathbb{Z}$ and every element in it other than the identity $1_{X}$ acts minimally on $X$. We call a space Slovakian if every homeomorphism other than $1_{X}$ is topologically transitive. Using such Slovakian spaces we construct a space $X$ such that $H(X)$ is topologically transitive on $X$, every element of $H(X)$ is chain 
transitive, and the homeomorphism group of the Cantor set occurs as a closed, topological subgroup of $H(X)$.

\section{Relation Dynamics}

It will be convenient to use the dynamics of closed relations, and so we briefly review the ideas from [4]. Recall that our spaces $X, Y$, etc. are assumed to be nonempty, compact, metrizable spaces with a fixed metric chosen when necessary.

For spaces $X, Y$, a relation $R: X \rightarrow Y$ is a subset of $X \times Y$. The set $R$ is a relation on $X$ when $Y=X$. A map is a relation such that $R(x)=\{y:(x, y) \in R\}$ is a singleton set for every $x \in X$. For $A \subset X$, the image $R(A)$ is defined to be $\bigcup_{x \in A} R(x)$. Equivalently, $R(A)$ is the projection to $Y$ of $R \cap(A \times Y) \subset X \times Y$. The inverse $R^{-1}: Y \rightarrow X$ is defined to be $\{(y, x):(x, y) \in R\}$. For $B \subset Y$, we let $R^{*}(B)=\{x \in$ $X: R(x) \subset B\}=X \backslash R^{-1}(Y \backslash B)$. So $R^{*}(B) \subset R^{-1}(B) \cup R^{*}(\emptyset)$. If $R$ is a map, then $R^{*}(B)=R^{-1}(B)$.

For example, $\bar{V}_{\epsilon}=\{(x, y) \in X \times X: d(x, y) \leq \epsilon\}$ is a relation on $X$ with $\bar{V}_{\epsilon}(x)$ the closed ball of radius $\epsilon$ and center $x$. When $\epsilon=0$, $\bar{V}_{\epsilon}=1_{X}$, the identity map on $X$.

If $R: X \rightarrow Y$ and $S: Y \rightarrow C$ are relations, then the composition $S \circ R: X \rightarrow C$ is the image under the projection to $X \times C$ of the set $(R \times C) \cap(X \times S) \subset X \times Y \times C$. Thus, $(x, c) \in S \circ R$ iff there exists $y \in Y$ such that $(x, y) \in R$ and $(y, c) \in S$. Composition is associative, and $(S \circ R)^{-1}=R^{-1} \circ S^{-1}$.

A relation $R$ on $X$ is reflexive when $1_{X} \subset R$, symmetric when $R^{-1}=$ $R$, and transitive when $R \circ R \subset R$.

For a relation $R$ on $X$, we let $R^{n+1}=R^{n} \circ R$ and $R^{-n}=\left(R^{-1}\right)^{n}$ for $n=1,2, \ldots$, and let $R^{0}$ be the identity $1_{X}$. We define the cyclic set $|R|=\{x:(x, x) \in R\}$.

For a relation $R$ on $X$ a subset $A$ of $X$ is called forward $R$-invariant (or $R$-invariant) if $R(A) \subset A$ (resp. $R(A)=A$ ).

For a relation $R$ on $X$, the orbit relation is $\mathcal{O} R=\bigcup_{n=1}^{\infty} R^{n}$, and the orbit closure relation $\mathcal{R} R$ is defined by $\mathcal{R} R(x)=\overline{\mathcal{O} R(x)}$ for all $x \in X$. The wandering relation is $\mathcal{N} R=\overline{\mathcal{O} R}$. Even when $R$ is a continuous map, $\mathcal{R} R$ is usually not closed and so is a proper subset of $\mathcal{N} R$.

The chain relation is

$$
\mathrm{e} R=\bigcap_{\epsilon>0} \mathcal{O}\left(\bar{V}_{\epsilon} \circ R \circ \bar{V}_{\epsilon}\right) .
$$

Both $\mathcal{O} R$ and $\mathcal{C} R$ are transitive relations. Since $\left(R^{n}\right)^{-1}=\left(R^{-1}\right)^{n}$, it follows that $\mathcal{O}\left(R^{-1}\right)=(\mathcal{O} R)^{-1}, \mathcal{N}\left(R^{-1}\right)=(\mathcal{N} R)^{-1}$ and $\mathcal{C}\left(R^{-1}\right)=$ 
$(\mathcal{C} R)^{-1}$. These operators on relations are monotone, i.e. they preserve inclusions, and $\mathcal{O}$ and $\mathcal{C}$ are idempotent. That is,

$$
\mathcal{O}(\mathcal{O} R)=\mathcal{O} R \quad \text { and } \quad \mathcal{C}(\mathcal{C} R)=\mathcal{C} R .
$$

It then follows that

$$
\begin{gathered}
\mathcal{R}(\mathcal{O} R)(x)=\overline{\mathcal{O}(\mathcal{O} R)(x)}=\overline{\mathcal{O} R(x)}=\mathcal{R} R(x), \\
\mathcal{N}(\mathcal{O} R)=\overline{\mathcal{O}(\mathcal{O} R)}=\overline{\mathcal{O} R}=\mathcal{N} R \\
\mathcal{C} R \subset \mathcal{C}(\mathcal{O} R) \subset \mathcal{C}(\mathcal{C} R)=\mathcal{C} R .
\end{gathered}
$$

If $R$ is a transitive relation on $X$, then $R \cap R^{-1}$ is a symmetric, transitive relation which restricts to an equivalence relation on $|R|$. We call the equivalence classes the basic sets of $R$.

A closed relation $R$ is a closed subset of $X \times Y$. A map is continuous iff it is a closed relation. The composition of closed relations is closed, and the image of a closed set by a closed relation is closed. So if $B$ is open in $Y$, then $R^{*}(B)$ is open in $X$. For any relation $R$, the extensions $\mathcal{N} R$ and $\mathcal{C} R$ are closed relations.

It is easy to see that $\mathrm{C} R=\mathrm{e} \bar{R}$, where $\bar{R}$ is the closure of $R$. If $R$ is a closed relation, then $\mathrm{C} R=\bigcap_{\epsilon>0} \mathcal{O}\left(\bar{V}_{\epsilon} \circ R\right)$, see [4] Proposition 1.18. If $R$ is a closed relation on $X$, then $|R|$ is a closed subset of $X$. If $R$ is a closed, transitive relation, the basic sets $\left\{R(x) \cap R^{-1}(x): x \in|R|\right\}$ are closed.

For a sequence $\left\{A_{n}\right\}$ of closed sets, $\limsup \left\{A_{n}\right\}=\bigcap_{n} \overline{\bigcup_{i \geq n} A_{i}}$. We have the identity $\overline{\bigcup_{n} A_{n}}=\left(\bigcup_{n} A_{n}\right) \cup \limsup \left\{A_{n}\right\}$. If $R$ is a closed relation on $X$ and $A$ is a closed subset of $X$, then we define $\omega R[A]=\lim \sup \left\{R^{n}(A)\right\}$.

If $A$ is forward $R$-invariant and $R$ is closed, then the sequence of closed sets $\left\{R^{n}(A)\right\}$ is decreasing and $\omega R[A]$ is the intersection. Furthermore, if $y \in \omega R[A]$, then $R^{-1}(y)$ meets every $\left\{R^{n}(A)\right\}$, and so by compactness it meets $\omega R[A]$ itself. It follows that $\omega R[A]$ is the maximum $R$-invariant subset of the closed, forward $R$-invariant set $A$.

If $R$ is closed, then for $x \in X$ we let

$$
\omega R(x)=\omega R[x]=\limsup \left\{R^{n}(x)\right\},
$$

defining the omega limit point relation $\omega R$ on $X$. We also define $\Omega R=$ $\lim \sup \left\{R^{n}\right\}$ for a general relation $R$, so $\Omega R$ is a closed relation. When $R$ is closed, we have the following identities:

$$
\mathcal{R} R=\mathcal{O} R \cup \omega R \quad \text { and } \quad \mathcal{N} R=\mathcal{O} R \cup \Omega R .
$$

Since $\mathcal{C} R$ is closed and transitive, the sequence $\left\{(\mathcal{C} R)^{n}\right\}$ is decreasing with intersection $\Omega \mathfrak{C} R=\omega \mathcal{C} R$. 
If $R$ is closed, then the following useful identities hold for the chain relation:

$$
\begin{aligned}
\mathfrak{C} R & =\mathcal{O} R \cup \Omega \mathcal{C} R, \\
R \cup(\mathcal{C} R) \circ R & =\mathcal{C} R=R \cup R \circ(\mathcal{C} R),
\end{aligned}
$$

see [4] Proposition 2.4 (c) and Proposition $1.11(\mathrm{~d})$.

It is not usually true that $(\omega R)^{-1}=\omega\left(R^{-1}\right)$. We write $\alpha R$ for $\omega\left(R^{-1}\right)$, defining the alpha limit point relation.

The points of $|\mathcal{O} R|$ are called periodic points for $R,|\mathcal{R} R|$ are the recurrent points, $|\mathcal{N} R|$ are the non-wandering points, and $|\mathcal{C} R|$ are the chain recurrent points. When $R$ is a closed relation, we can apply the inclusion $|\mathcal{O} R| \subset|\omega R|$ to (2.4) and (2.5) to obtain $|\mathcal{R} R|=|\omega R|$, $|\mathcal{N} R|=|\Omega R|$ and $|\mathcal{C} R|=|\Omega \mathcal{C} R|$. We call $x$ a transitive point for $R$ when $\mathcal{R} R(x)=X$. We denote by $\operatorname{Trans}(R)$ the (possibly empty) set of transitive points.

On $|\mathcal{C} R|, \mathcal{C} R \cap \mathcal{C} R^{-1}$ is a closed equivalence relation. We call the equivalence classes, i.e. the basic sets for $\mathcal{C} R$, the chain components of $R$.

For a relation $R$ on $X$, we will say that $R$ is minimal when $\mathcal{R} R=$ $X \times X$, topologically transitive when $\mathcal{N} R=X \times X$, and chain transitive when $\mathcal{C} R=X \times X$. We call $R$ central when $|\mathcal{N} R|=X$ and chain recurrent when $|\mathrm{e} R|=X$. From (2.3) it follows that any one of these properties holds for $R$ iff it holds for $\mathcal{O} R$.

Observe that if $R$ is minimal, then $X$ contains no proper closed, forward $R$-invariant subset. If $R$ is a continuous map, then the converse is true as well since $\omega R(x)$ is then $R$-invariant and nonempty for every $x$. Thus, if $R$ is a continuous map, it is minimal iff $X=\operatorname{Trans}(R)$.

A set $U$ is inward for a closed relation $R$ on $X$ if $U$ is closed and $R(U) \subset U^{\circ}$, the interior of $U$. Since $R(U)$ has a positive distance from the complement of $U$, it easily follows that $U$ is inward for $\mathcal{C} R$ when it is inward for $R$. An inward set is therefore forward $\mathcal{C} R$-invariant. In general, a closed set $A$ is forward $\mathcal{C} R$-invariant iff it has a neighborhood base consisting of inward sets, see [4] Theorem 3.3(c). If $U$ is an inward set, then $A_{+}=\omega R[U]=\bigcap_{n \in \mathbb{N}} R^{n}(U)$ is the associated attractor. The set $X \backslash U^{\circ}$ is inward for $R^{-1}$, and $A_{-}=\omega\left(R^{-1}\right)\left[X \backslash U^{\circ}\right]$ is the dual repellor for $R$. The pair $A_{+}, A_{-}$is called an attractor-repellor pair. If

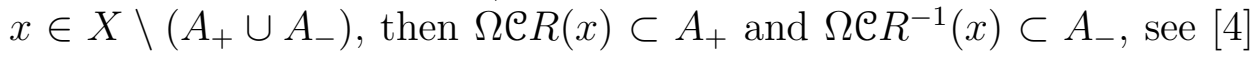
Proposition 3.9.

Notice that a clopen set is inward iff it is forward invariant. If an inward set is invariant, then it is clopen.

Proposition 2.1. Let $R$ be a closed relation on $X$. 
CHAIN TRANSITIVE HOMEOMORPHISMS ON A SPACE: ALL OR NONE 9

(a) If $A_{+}, A_{-}$is an attractor-repellor pair, then $|\mathcal{C} R| \subset A_{+} \cup A_{-}$ and the intersections $A_{+} \cap|\mathcal{C} R|$ and $A_{-} \cap|\mathcal{C} R|$ are each clopen in $|\mathrm{e} R|$.

(b) If $x \in X \backslash|\mathcal{C} R|$, then there exists an attractor-repellor pair $A_{+}, A_{-}$such that $x \notin A_{+} \cup A_{-}$.

(c) If $x, y \in|\mathrm{C} R|$, then $y \in \mathcal{C} R(x)$ iff, for all attractors $A, x \in A$ implies $y \in A$.

(d) The space of chain components, i.e. the quotient space of $|\mathrm{C} R|$ by the equivalence relation $\mathrm{e} R \cap \mathrm{e} R^{-1}$, is a compact zero-dimensional metric space.

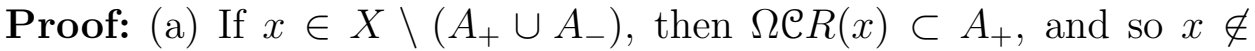
$\Omega \mathcal{C} R(x)$. If $U$ is an inward set with $\omega R[U]=A_{+}$, then $U^{\circ} \cap|\mathrm{e} R|=$ $A_{+} \cap|\mathrm{e} R|$.

(b), (c) These are part of Proposition 3.11 of [4].

(d) From (b) and (c) applied to $R$ and $R^{-1}$ it follows that every chain component $C$ is the intersection of $\{A \cap|\mathrm{C} R|: A$ is an attractor or repellor and $C \subset A\}$. Hence, the attractors and repellors induce a clopen subbase on the space of chain components.

For a relation $R$ on a space $X$ we say that $X$ is $R$-decomposable if there is a proper, forward $R$-invariant decomposition $\left\{A_{1}, A_{2}\right\}$ of $X$, i.e. each is a nonempty, closed, forward $R$-invariant subset and $A_{1} \cap A_{2}=$ $\emptyset, A_{1} \cup A_{2}=X$. Such a pair of proper clopen sets is called an $R$ decomposition. Since the sets are clopen and forward $R$-invariant, they are inward for $R$. Hence, an $R$-decomposition is a $\mathcal{C} R$-decomposition. So the notion of decomposability is the same for $R, \mathcal{O} R, \mathcal{R} R, \mathcal{N} R$ and e R.

If no such a decomposition exists, $X$ is said to be $R$-indecomposable.

For any relation $R$ on $X$ let $R_{ \pm}=R \cup 1_{X} \cup R^{-1}$. This is a reflexive and symmetric relation on $X$ so that $\mathcal{O}\left(R_{ \pm}\right)$and $\mathcal{C}\left(R_{ \pm}\right)$are equivalence relations on $X$.

Proposition 2.2. For a relation $R$ on $X$, the following conditions are equivalent:

(i) The space $X$ is $R$-indecomposable.

(ii) The space $X$ is $R_{ \pm}$-indecomposable.

(iii) The relation $R_{ \pm}$is chain transitive.

Proof: (i) $\Leftrightarrow$ (ii): If a set is forward $R$-invariant, then its complement is forward $R^{-1}$-invariant. Hence, an $R$-decomposition is an $R_{ \pm^{-}}$ decomposition. Clearly, the reverse is true since $R \subset R_{ \pm}$. 
(iii) $\Rightarrow$ (ii): If $\left\{A_{1}, A_{2}\right\}$ is a decomposition, then each is inward for $R_{ \pm}$and therefore forward $\mathcal{C} R_{ \pm}$-invariant. Hence, $R_{ \pm}$is not chain transitive.

(ii) $\Rightarrow$ (iii): If $R_{ \pm}$is not chain transitive, then the equivalence relation $\mathrm{e} R_{ \pm}$on $X$ has more than one equivalence class. By Proposition 2.1 (d) the space of equivalence classes is zero-dimensional. Hence, there is a pair of disjoint, nonempty, clopen sets $\left\{A_{1}, A_{2}\right\}$ which cover $X$ such that each is a union of equivalence classes. Since each equivalence class is $\mathcal{C} R_{ \pm}$-invariant, it follows that $\left\{A_{1}, A_{2}\right\}$ is a $R_{ \pm}$-decomposition.

Definition 2.3. Let $\pi: X_{1} \rightarrow X_{2}$ be a continuous map between spaces, and let $R_{i}$ be a relation on $X_{i}$ for $i=1,2$. We say that $\pi$ maps $R_{1}$ to $R_{2}$ if $\pi \circ R_{1} \subset R_{2} \circ \pi$ and that $\pi$ is a semi-conjugacy from $R_{1}$ to $R_{2}$ if $\pi \circ R_{1}=R_{2} \circ \pi$.

Proposition 2.4. Let $\pi: X_{1} \rightarrow X_{2}$ be a continuous map between spaces, and let $R_{i}$ be a relation on $X_{i}$ for $i=1,2$.

(a) The function $\pi$ maps $R_{1}$ to $R_{2}$ iff $(\pi \times \pi)\left(R_{1}\right) \subset R_{2}$. If $\pi$ is a semi-conjugacy from $R_{1}$ to $R_{2}$ and $\pi\left(X_{1}\right)=X_{2}$, i.e. $\pi$ is surjective, then $(\pi \times \pi)\left(R_{1}\right)=R_{2}$.

(b) If $R_{i}$ is a mapping for $i=1,2$, then $\pi$ is a semi-conjugacy from $R_{1}$ to $R_{2}$ if it maps $R_{1}$ to $R_{2}$.

(c) If $\pi$ maps $R_{1}$ to $R_{2}$, then $\pi$ maps $R_{1}^{n}$ to $R_{2}^{n}$ for all $n \in \mathbb{Z}$ and maps $\mathcal{A} R_{1}$ to $\mathcal{A} R_{2}$ for $\mathcal{A}=\mathcal{O}, \mathcal{R}, \mathcal{N}$ and $\mathcal{C}$.

(d) Assume $\pi$ is surjective and maps $R_{1}$ to $R_{2}$. If $R_{1}$ is minimal, topologically transitive, central, chain transitive or chain recurrent, then $R_{2}$ satisfies the corresponding property.

(e) If $\pi$ is a semi-conjugacy from $R_{1}$ to $R_{2}$ and $\pi$ is an open map, then $\pi$ is a semi-conjugacy from $\mathcal{A} R_{1}$ to $\mathcal{A} R_{2}$ for $\mathcal{A}=\mathcal{O}, \mathcal{R}, \mathcal{N}$ and $\mathrm{C}$.

Proof: (a) Firstly, $\pi$ maps the relation $R_{1}$ to $R_{2}$ iff $(x, y) \in R_{1}$ implies $(\pi(x), \pi(y)) \in R_{2}$, and so the first equivalence is clear. The second result is easy to check.

(b) An inclusion between functions is an equation.

(c) Given $\epsilon>0$, there exists $\delta>0$ such that $(\pi \times \pi)\left(\bar{V}_{\delta}\right) \subset V_{\epsilon}$ since $\pi$ is uniformly continuous. If $\left\{\left(x_{n}, y_{n}\right) \in R_{1}\right\}$ is a finite or infinite sequence with $\left(y_{n}, x_{n+1}\right) \in \bar{V}_{\delta}$, then $\left\{\left(\pi\left(x_{n}\right), \pi\left(y_{n}\right)\right) \in R_{2}\right\}$ with $\left(\pi\left(y_{n}\right), \pi\left(x_{n+1}\right)\right) \in \bar{V}_{\epsilon}$. That is, $\delta$-chains for $R_{1}$ are mapped to $\epsilon$-chains for $R_{2}$. It follows that $\pi$ maps $\mathcal{C} R_{1}$ to $\mathcal{C} R_{2}$. The others are easy to check.

(d) If $\pi$ is surjective and maps $\mathcal{A} R_{1}$ to $\mathcal{A} R_{2}$, then $X_{1} \times X_{1}=\mathcal{A} R_{1}$ implies $X_{2} \times X_{2}=\mathcal{A} R_{2}$, and $1_{X_{1}} \subset \mathcal{A} R_{1}$ implies $1_{X_{2}} \subset \mathcal{A} R_{2}$. 
(e) The function $1_{X_{1}} \times \pi$ maps $1_{X_{1}}$ to $\pi \subset X_{1} \times X_{2}$. If $\pi$ is open and $\epsilon>0$, then $1_{X_{1}} \times \pi$ maps $\bar{V}_{\epsilon}$ to a neighborhood of $\pi$ and so contains $\bar{V}_{\delta} \circ \pi$ for some $\delta>0$. That is, $\bar{V}_{\delta} \circ \pi \subset \pi \circ \bar{V}_{\epsilon}$. If $\left\{\left(u_{n}, v_{n}\right) \in R_{2}\right\}$ is a finite or infinite sequence with $\left(v_{n}, u_{n+1}\right) \in \bar{V}_{\delta}$ and $\pi\left(x_{n}\right)=u_{n}$, then because $\pi$ is a semi-conjugacy on $R_{1}$, there exists $y_{n} \in X_{1}$ such that $\left(x_{n}, y_{n}\right) \in R_{1}$ and $\pi\left(y_{n}\right)=v_{n}$, and there exists $x_{n+1}$ such that $\left(y_{n}, x_{n+1}\right) \in \bar{V}_{\epsilon}$ and $\pi\left(x_{n+1}\right)=u_{n+1}$. Thus, every $\delta$-chain for $R_{2}$ can be lifted to an $\epsilon$-chain for $R_{1}$ with a given initial lift. That is, $\pi$ is a semiconjugacy from $\mathcal{e} R_{1}$ to $\mathcal{C} R_{2}$. The cases of $\mathcal{A}=\mathcal{O}, \mathcal{R}$ and $\mathcal{N}$ are similar. In fact, for $\mathcal{O}$ and $\mathcal{R}$ it is not necessary that the map be open.

Remark: Suppose $R_{i}=1_{X_{i}}$ with $X_{2}=[0,1]$ and $X_{1}=\{-1\} \cup[0,1]$, and let $\pi: X_{1} \rightarrow X_{2}$ be an extension of the identity on $[0,1]$, mapping -1 to some point of $[0,1]$. The map $\pi$ is a semi-conjugacy from $R_{1}$ to $R_{2}$ and maps $\mathcal{C} R_{1}$ onto $\mathcal{C} R_{2}$, but it is not a semi-conjugacy from $\mathcal{C} R_{1}$ to $\mathrm{e} R_{2}$.

As indicated in the Introduction, our concern is with homeomorphisms. However, we will apply this machinery to three different relations.

Let $H(X)$ be the homeomorphism group of $X$. For $f \in H(X)$, we define $f_{ \pm}$as the closed relation $f \cup 1_{X} \cup f^{-1}$. Clearly, we have

$$
\begin{aligned}
\mathcal{O} f & =\left\{f^{n}: n \in \mathbb{N}\right\}, \\
\mathcal{O} f_{ \pm} & =\left\{f^{n}: n \in \mathbb{Z}\right\}=\mathcal{O} f \cup 1_{X} \cup \mathcal{O} f^{-1}, \\
\mathcal{R} f(x) & =\overline{\left\{f^{n}(x): n \in \mathbb{N}\right\}}, \\
\mathcal{R} f_{ \pm}(x) & =\overline{\left\{f^{n}(x): n \in \mathbb{Z}\right\}} \text { for } x \in X, \\
\mathcal{N} f_{ \pm} & =\mathcal{N} f \cup 1_{X} \cup \mathcal{N} f^{-1}, \\
\mathcal{R} f_{ \pm} & =\mathcal{R} f \cup 1_{X} \cup \mathcal{R}\left(f^{-1}\right) .
\end{aligned}
$$

On the other hand, $\mathcal{e} f_{ \pm}$is in general larger than $\mathcal{e} f \cup 1_{X} \cup \mathcal{C} f^{-1}$. The latter relation need not be transitive. See Example 2.8 below.

Since $1_{X} \subset \mathcal{O} f_{ \pm}$, every point is recurrent for $f_{ \pm}$.

An action of a group $G$ on $X$ is a homomorphism $\rho: G \rightarrow H(X)$. If $G$ is a subgroup of $H(X)$, then $G$ acts on $X$ by evaluation. That is, $\rho$ is the inclusion. With the action understood we let $h_{G}=\bigcup\{f \in \rho(G)\}$. This is just the orbit relation of the action of $G$ on $X$. It is an equivalence relation but usually not closed. Since it is an equivalence relation, $\mathcal{O} h_{G}=h_{G}$ and $\mathcal{N} h_{G}=\overline{h_{G}}$. Since $h_{G}$ is reflexive, every point is recurrent for $h_{G}$.

If $G$ is the cyclic group generated by a homeomorphism $f$, then $h_{G}=\mathcal{O} f_{ \pm}$. 
When $G=H(X)$, we write $h_{X}$ for $h_{G}$. Clearly, $H(X)$-decomposability as described in the Introduction is just $h_{X}$-decomposability.

For a space $X$, let $\operatorname{Iso}(X)$ denote the set of isolated points of $X$.

Proposition 2.5. Let $f \in H(X)$ and $\rho: G \rightarrow H(X)$ be an action of a group $G$ on $X$. Let $\mathcal{B}$ be a countable base of nonempty open sets for $X$, and let $\mathcal{B}^{*}$ be the collection of finite covers of $X$ by elements of $\mathcal{B}$.

(a) The homeomorphism $f$ is central iff the $G_{\delta}$ set of recurrent points, $|\omega f|$, is dense.

$$
|\omega f|=\bigcap_{\mathcal{A} \in \mathcal{B}^{*}, n \in \mathbb{N}} \bigcup_{U \in \mathcal{A}, i \geq n}\left\{U \cap f^{-i}(U)\right\} .
$$

If $f$ is central, then any isolated point $x$ of $X$ is a periodic point for $f$.

(b) The homeomorphism $f$ is topologically transitive iff the set of transitive points, Trans $(f)$, is nonempty, in which case

$$
\begin{aligned}
\operatorname{Trans}(f) & =\{x: \omega f(x)=X\} \\
& =\bigcap_{U \in \mathcal{B}, n \in \mathbb{N}} \bigcup_{i \geq n}\left\{f^{-i}(U)\right\}
\end{aligned}
$$

is a dense $G_{\delta}$ subset of $X$. If $f$ is topologically transitive, then $f^{-1}$ is topologically transitive. If $f$ is topologically transitive, then $X$ is perfect or consists of a single periodic orbit for $f$.

(c) The relation $f_{ \pm}$is topologically transitive iff the set of transitive points, $\operatorname{Trans}\left(f_{ \pm}\right)$, is nonempty, in which case

$$
\operatorname{Trans}\left(f_{ \pm}\right)=\bigcap_{U \in \mathcal{B}} \bigcup_{n \in \mathbb{Z}}\left\{f^{-n}(U)\right\}
$$

is a dense $G_{\delta}$ subset of $X$. If $f_{ \pm}$is topologically transitive and $x$ is an isolated point of $X$, then

$$
\operatorname{Trans}\left(f_{ \pm}\right)=\mathcal{O} f_{ \pm}(x)=\operatorname{Iso}(X) .
$$

Thus, if $f_{ \pm}$is topologically transitive, then either $\operatorname{Iso}(X)=\emptyset$, so $X$ is perfect, or else $\operatorname{Iso}(X)$ is dense. If $\operatorname{Iso}(X)$ is finite and nonempty, then $X$ consists of a single periodic orbit.

(d) The following are equivalent:

(i) $f$ is topologically transitive;

(ii) $f_{ \pm}$is topologically transitive and $f$ is central;

(iii) $f_{ \pm}$is topologically transitive and $X$ is either perfect or consists of a single periodic orbit for $f$.

In that case, $\operatorname{Trans}\left(f_{ \pm}\right)=\operatorname{Trans}(f) \cup \operatorname{Trans}\left(f^{-1}\right)$. 
(e) The relation $h_{G}$ is topologically transitive iff the set of transitive points, $\operatorname{Trans}\left(h_{G}\right)$, is nonempty, in which case

$$
\operatorname{Trans}\left(h_{G}\right)=\bigcap_{U \in \mathcal{B}} \bigcup_{f \in G}\left\{f^{-1}(U)\right\}
$$

is a dense $G_{\delta}$ subset of $X$.

Proof: For a relation $R$ on $X$ and subsets $U, V \subset X$, let

$$
\begin{aligned}
N_{R}(U, V) & =\left\{n \in \mathbb{N}: R^{n}(U) \cap V \neq \emptyset\right\} \\
& =\left\{n \in \mathbb{N}: U \cap R^{-n}(V) \neq \emptyset\right\} .
\end{aligned}
$$

Clearly, $R$ is central iff $N_{R}(U, U) \neq \emptyset$ for all nonempty open subsets $U$, and $R$ is topologically transitive iff $N_{R}(U, V) \neq \emptyset$ for all nonempty open subsets $U, V$. If $R=f \in H(X)$ and $N_{f}(U, V)$ is finite for some open $U, V$, then with $n=\max N_{f}(U, V)$ we let $W=U \cap f^{-n}(V)$. Then $W$ is a nonempty open set and $N_{f}(W, V)=\emptyset$. Hence, if $f$ is central, then $N_{f}(U, U)$ is infinite, and if $f$ is topologically transitive, then $N_{f}(U, V)$ is infinite for all nonempty open $U, V$.

The equations (2.7)-(2.13) are easy to check, and density follows from the Baire Category Theorem.

Now assume that $f_{ \pm}$is topologically transitive and that $x$ is an isolated point of $X$. If $V$ is a nonempty open set, then $N_{f_{ \pm}}(\{x\}, V)=\emptyset$ unless $V$ meets the orbit $\mathcal{O} f_{ \pm}(x)$. Hence, $x \in \operatorname{Trans}\left(f_{ \pm}\right)$. If $y$ is another isolated point, then $N_{f_{ \pm}}(\{x\},\{y\}) \neq \emptyset$ implies that $y$ is in the $f_{ \pm}$orbit of $x$. Thus, (2.11) holds. In particular, Iso $(X)$ is dense if it is nonempty. If it is finite and nonempty, then $X=\operatorname{Iso}(X)$ because the latter is closed and dense. Since the elements of Iso $(X)$ lie on a single orbit, $X$ consists of a single periodic orbit.

If $f$ is central and $x \in \operatorname{Iso}(X)$, then $N_{f}(\{x\},\{x\}) \neq \emptyset$ iff $x$ is a periodic point. Thus, if $f_{ \pm}$is transitive and $f$ is central, then either $X$ is perfect or it consists of a single periodic orbit, proving the implication (ii) $\Rightarrow$ (iii) in (d). Since (i) obviously implies (ii) in (d), it follows that if $f$ is topologically transitive, then either $X$ is perfect or consists of a single periodic orbit. So if $\mathcal{R} f(x)=X$, then $\overline{\left\{f^{i}(x): i \geq n\right\}}=X$ for every $n \in \mathbb{N}$. Intersecting, we see that $\omega f(x)=X$. Thus, $\operatorname{Trans}(f)=$ $\{x: \omega f(x)=X\}$.

Finally, if $X$ is a single periodic orbit, then $f$ is topologically transitive and every point is a transitive point for $f$ and $f^{-1}$. Now assume that $X$ is perfect and that $f_{ \pm}$is transitive. If $x \in \operatorname{Trans}\left(f_{ \pm}\right)$, then $\overline{\left\{f^{i}(x): i \in \mathbb{Z},|i| \geq n\right\}}=X$ for all $n \in \mathbb{N}$. Intersecting, we obtain that $\omega f(x) \cup \alpha f(x)=X$. In particular, $x$ is in one of these. If $x$ is contained in a closed invariant set $A$ like $\omega f(x)$ or $\alpha f(x)$, then 
$X=\mathcal{R}\left(f_{ \pm}\right)(x) \subset A$. Thus, $x \in \operatorname{Trans}(f) \cup \operatorname{Trans}\left(f^{-1}\right)$, so either $f$ or $f^{-1}$ is topologically transitive. But $\mathcal{N}\left(f^{-1}\right)=(\mathcal{N} f)^{-1}$ implies that $f^{-1}$ is topologically transitive if $f$ is.

Remark: There are various, slightly conflicting, definitions of topological transitivity. These are sorted out in [6]. We are following [4].

Lemma 2.6. (a) Let $f \in H(X)$ and $x \in X$. The sets $\omega f(x)$ and $\alpha f(x)$ are $f$-invariant. If $x \in|\mathcal{C} f|$, then $\mathcal{C} f(x)$ and $\mathcal{C} f^{-1}(x)$ are $f$-invariant.

(b) The chain components of $1_{X}$ are the components of $X$.

Proof: (a) For a bijection $f$ on $X$, a subset $A$ is $f$-invariant iff it is $f^{-1}$-invariant iff it is forward invariant for both $f$ and $f^{-1}$.

When $f$ is a continuous map on $X$, then $y \in \omega f(x)$ when there is a subsequence $\left\{f^{n_{i}}(x)\right\}$ of the orbit sequence which converges to $y$. Then $\left\{f^{n_{i}+1}(x)\right\}$ converges to $f(y)$, and if a subsequence of $\left\{f^{n_{i}-1}(x)\right\}$ converges to $z$, then $f(z)=y$. That is, $\omega f(x)$ is a nonempty, closed, $f$-invariant subset of $X$ when $f$ is a continuous map on $X$. So when $f$ is a homeomorphism, the same is true for $\alpha f(x)$.

For $f \in H(X)$ we obtain from the identity (2.5) that $1_{X} \cup \mathfrak{e} f=f^{-1}$ 。 $\mathcal{C} f$. If $x \in \mathcal{C} f(x)$, then $\mathcal{C} f(x)=\{x\} \cup \mathcal{C} f(x)=f^{-1}(\mathcal{C} f(x))$. That is, $\mathcal{e} f(x)$ is $f^{-1}$ invariant and so is $f$ invariant. Since $\mathcal{C}\left(f^{-1}\right)=(\mathcal{C} f)^{-1}$ the same is true for $\mathcal{C} f^{-1}(x)$.

(b) The space of chain components being zero-dimensional by Proposition $2.1(\mathrm{~d})$, every connected set contained in $|\mathcal{C} R|$ is entirely contained in a single chain component. In particular, when $R$ is $1_{X}$, every component is a subset of a chain component. On the other hand, when $R=1_{X}$, every clopen subset is inward for $R$ and so contains any chain component that it meets. It follows that the components are the chain components.

Proposition 2.7. Let $f \in H(X)$ and $\rho: G \rightarrow H(X)$ be an action of a group $G$ on $X$.

(a) The following are equivalent:

(i) $X$ is $f$-indecomposable;

(ii) $X$ is $f_{ \pm}$-indecomposable;

(iii) $f_{ \pm}$is chain transitive.

(b) The relation $h_{G}$ is chain transitive iff $h_{G}$ is indecomposable.

(c) If $f$ is chain recurrent, then $\mathrm{e} f=\mathrm{e}\left(f_{ \pm}\right)$.

(d) The following are equivalent:

(i) $f$ is chain transitive;

(ii) $f$ is chain recurrent and $f_{ \pm}$is chain transitive;

(iii) $f$ is chain recurrent and indecomposable. 
(e) If $X$ is connected, then $f$ is chain transitive iff it is chain recurrent. In particular, $1_{X}$ is chain transitive.

Proof: (a), (b) Since $h_{G}=\left(h_{G}\right)_{ \pm}$, these results are a special cases of Proposition 2.2 applied with $R=f$ and $R=h_{G}$.

(c) If $f$ is chain recurrent, then $|\mathcal{e} f|=X$ and $1_{X} \subset \mathcal{e} f$. For any $x \in X$, we have $x \in \mathcal{C} f(x)$, and this set is $f$-invariant by Lemma 2.6. Hence, $f^{-1}(x) \in \mathcal{C} f(x)$. Thus, $f^{-1} \subset \mathcal{C} f$. Since $f \subset f_{ \pm} \subset \mathcal{C} f$, it follows from (2.2) that $\mathcal{C} f \subset \mathcal{e}\left(f_{ \pm}\right) \subset \mathcal{e} \mathcal{C} f=\mathcal{C} f$.

(d) If $f$ is chain transitive, then it is clearly chain recurrent, and $f \subset f_{ \pm}$implies that $f_{ \pm}$is chain transitive. The converse follows from (c). This proves the equivalence of (i) and (ii). The equivalence of (ii) and (iii) follows from (a).

(e) If $X$ is connected, then by Lemma 2.7(b) $X$ consists of a single chain component for $1_{X}$, and so $1_{X}$ is chain transitive. So if $f$ is chain recurrent, then $X \times X=\mathcal{e} 1_{X} \subset \mathcal{e} f=\mathcal{e} f$ by (2.2) again.

Example 2.8. On $\mathbb{Z}$ let $t$ be the translation bijection given by $n \mapsto$ $n+1$. Let $\mathbb{Z}^{*}$ be the one-point compactification adjoining the point $\pm \infty$ to $\mathbb{Z}$, and let $\mathbb{Z}^{* *}$ be the two-point compactification adjoining the points $-\infty,+\infty$ to $\mathbb{Z}$. Let $t^{*}$ and $t^{* *}$ be the homeomorphisms extending $t$ to $\mathbb{Z}^{*}$ and $\mathbb{Z}^{* *}$, respectively. Both $t_{ \pm}^{*}$ and $t_{ \pm}^{* *}$ are topologically transitive with $\mathbb{Z}$ the orbit of isolated points, and so $\mathbb{Z}=\operatorname{Trans}\left(t_{ \pm}^{*}\right)=\operatorname{Trans}\left(t_{ \pm}^{* *}\right)$. Of course, both $t_{ \pm}^{*}$ and $t_{ \pm}^{* *}$ are chain transitive, but $t^{*}$ is also chain transitive while $t^{* *}$ is not.

Let $X$ be the quotient space of $Z^{* *} \times\{0,1\}$ with the fixed points $(+\infty, 0)$ and $(+\infty, 1)$ identified. Let $f$ be the homeomorphism on $X$ induced by $t^{* *} \times 1_{\{0,1\}}$. Clearly, $f_{ \pm}$is chain transitive. But $\mathcal{C} f \cup 1_{X} \cup \mathcal{C} f^{-1}$ is contained in the image of $\left(Z^{* *} \times\{0\}\right)^{2} \cup\left(Z^{* *} \times\{1\}\right)^{2}$ in $X^{2}$ and so is a proper subset of $\mathcal{C}\left(f_{ \pm}\right)=X^{2}$. Furthermore, it is easy to check that in this case $\mathcal{C}\left(f \cup 1_{X}\right)=(\mathcal{C} f) \cup 1_{X}$. Hence, $R=f \cup 1_{X}$ is a closed relation such that $R$ is chain recurrent and $R_{ \pm}$is chain transitive, but $R$ is not chain transitive. Thus, Proposition 2.7 (c) and (d) do not extend to general relations.

Finally, we recall the Uniqueness of Cantor: any compact, perfect, zero-dimensional, metrizable space is homeomorphic to the Cantor set in $[0,1]$. We will call any such space a Cantor set. We will need the following well-known result, providing a brief sketch of the proof.

Proposition 2.9. For any space $X$ there exists a surjective continuous map $\pi: C \rightarrow X$ with $C$ a Cantor set.

Proof: With $\mathcal{B}$ a countable basis for $X$, let $Z$ be the closure in $X \times$ $\{0,1\}^{\mathcal{B}}$ of the set of pairs $\left\{(x, z): z_{U}=1 \Leftrightarrow x \in U\right\}$. The projection 
to $X$ is clearly onto, and because $\mathcal{B}$ is a basis, the projection to $\{0,1\}^{\mathcal{B}}$ is easily seen to be injective. It follows that $Z$ is compact and zerodimensional. If $C_{0}$ is a Cantor set, then $C=Z \times C_{0}$ is perfect as well as zero-dimensional and so is a Cantor set. Let $\pi$ be the composition of projections $C \rightarrow Z \rightarrow X$.

\section{Spaces which admit Chain Transitive Maps}

We begin with the relationship between omega limit sets and chain transitive subsets which was described in the introduction.

Proposition 3.1. If $f$ is a homeomorphism on a space $X$ and $x \in X$, then $\omega f(x)$ and $\alpha f(x)$ are chain transitive subsets, i.e. the restriction of $f$ to each of these nonempty, closed, invariant sets is chain transitive.

Proof: Let $y, y^{\prime} \in \omega f(x)$ and let $\epsilon>0$. Choose $\delta>0$ an $\epsilon / 2$-modulus of uniform continuity for $f$ with $\delta<\epsilon / 2$. There exists $N \in \mathbb{N}$ such that $n \geq N$ implies $f^{n}(x) \in \bar{V}_{\delta}(\omega f(x))$. There exist $n \geq N$ such that $d\left(f^{n}(x), y\right)<\delta$ and $k \in \mathbb{N}$ such that $d\left(f^{n+k}(x), y^{\prime}\right)<\delta$. For $i=0, \ldots, k$ choose $y_{i} \in \omega f(x)$ such that $d\left(f^{n+i}(x), y_{i}\right) \leq \delta$ with $y_{0}=y, y_{k}=y^{\prime}$. Hence, $d\left(f^{n+i+1}(x), f\left(y_{i}\right)\right) \leq \epsilon / 2$ and so $d\left(y_{i+1}, f\left(y_{i}\right)\right) \leq \epsilon$. That is, $\left\{y_{i}\right\}$ is an $\epsilon$-chain from $y$ to $y^{\prime}$. It follows that $\omega f(x)$ is a chain transitive subset. Applying the result to $f^{-1}$, we see that $\alpha f(x)=\omega\left(f^{-1}\right)(x)$ is a chain transitive subset as well.

We prove, conversely, in Theorem 3.11 (a) below that a chain transitive homeomorphism is the restriction to an omega limit set of a homeomorphism in a larger system.

In the constructions which follow, we will repeatedly use the process of attachment. Assume that $A$ is a nonempty, closed, nowhere dense subset of a space $X$ and that $h: A \rightarrow B$ is a continuous surjection. We may assume that $X$ and $B$ are disjoint. Otherwise, replace $X$ by $X \times\{0\}$ and $B$ by $B \times\{1\}$. Define $X / h$, the space with $X$ attached to $B$ via $h$ as follows: Let $\widetilde{h}$ denote the continuous retraction $h \cup 1_{B}$ : $A \cup B \rightarrow B$. Let $E_{h}=1_{X} \cup\left(\widetilde{h}^{-1} \circ \widetilde{h}\right)=1_{X} \cup(\widetilde{h} \times \widetilde{h})^{-1}\left(1_{B}\right)$, a closed equivalence relation on $X \cup B$. Let $X / h$ be the quotient space with projection $q_{h}: X \cup B \rightarrow X / h$. Since $q_{h}$ is injective on $B$, we may regard it as an identification and so regard $B$ as a subset of $X / h$. Furthermore, $q_{h}$ restricts to a surjection $X \rightarrow X / h$ which maps $A$ onto $B$ via $h$ and which is a homeomorphism between the dense open sets $X \backslash A \subset X$ and $(X / h) \backslash B \subset X / h$. When $B$ is a singleton, we write $q_{A}: X \rightarrow X / A$ for the quotient map and describe the result as smashing $A$ to a point. 
We will use some results of E. R. Lorch from [18] and [19] (see also [22]), which we will briefly review.

For a locally compact space $W$, a compactification of $W$ is a compact space $Y$ together with a dense embedding of $W$ into $Y$, i.e. a homeomorphism of $W$ onto a dense subset of $Y$. Because $W$ is locally compact, its image is an open, dense subset of $Y$, so $X=Y \backslash W$ is a nowhere dense, closed subset of $Y$. Reversing the point of view, we call $Y$ an extension of $X$ if $Y$ is a compact space and $X$ is a closed, nowhere dense subset of $Y$.

By a pair of spaces $(Y, X)$ we will mean a space and a closed subset, respectively. Recall our default assumption that a space is a nonempty, compact, metrizable space. We will call $(Y, X)$ an extension pair when $Y$ is an extension of $X$, i.e. when $X$ is nowhere dense in $Y$.

A continuous map $f: Y_{1} \rightarrow Y_{2}$ is a map of pairs $f:\left(Y_{1}, X_{1}\right) \rightarrow$ $\left(Y_{2}, X_{2}\right)$ when $f\left(X_{1}\right) \subset X_{2}$. If $X_{1}=X_{2}=X$, we will say that $f$ : $\left(Y_{1}, X\right) \rightarrow\left(Y_{2}, X\right)$ is a map rel $X$ if it restricts to the identity on $X$ and if, in addition, $f\left(Y_{1} \backslash X\right) \subset Y_{2} \backslash X$. These conditions imply that $1_{X}=(f \times f)^{-1}\left(1_{X}\right)$. So by intersecting over $\delta>0$ and using compactness, we see that for every $\epsilon>0$ there exists $\delta>0$ such that,

$$
\begin{gathered}
\text { if }(u, v) \in Y_{1} \times Y_{1} \text { and } \\
d_{2}(f(u), f(v)), d_{2}(f(u), X), d_{2}(f(v), X) \leq \delta \text {, then } \\
(u, v) \in \bar{V}_{\epsilon}^{d_{1}} \text {, and so } f^{-1}\left(\bar{V}_{\delta}^{d_{2}}(x)\right) \subset \bar{V}_{\epsilon}^{d_{1}}(x) \text { for all } x \in X,
\end{gathered}
$$

where $d_{1}, d_{2}$ are metrics on $Y_{1}$ and $Y_{2}$. Note that in considering different extensions $Y_{1}, Y_{2}$ of the same space $X$ we do not assume that the metrics $d_{1}$ and $d_{2}$ agree on $X$, although they are, of course, uniformly equivalent on $X$.

Definition 3.2. We call $X^{i}$ an isolated point extension of $X$, or just a point extension of $X$, if $X^{i}$ is an extension of $X$ with each point of $X^{i} \backslash X$ isolated. We then call $\left(X^{i}, X\right)$ a point extension pair.

A pair $(Y, A)$ is a point extension pair iff $Y$ is infinite, Iso $(Y)$ is dense and $A=Y \backslash \operatorname{Iso}(Y)$. Since $Y$ is separable, Iso $(Y)$ is denumerable, and so Lorch uses the term denumerable extension instead of point extension. Thus, $Y$ is a compactification of the denumerable discrete set $\operatorname{Iso}(Y)$.

For a point extension $\left(X^{i}, X\right)$, we define a canonical retraction $r$ : $X^{i} \rightarrow X$ so that for all $x \in X^{i}$

$$
d(x, r(x))=d(x, X) .
$$

The choice depends on the metric. Even for a fixed metric there may be more than one choice of point $r(x)$ which satisfies (3.2). For each $x$ 
we fix a choice to define $r$. Clearly,

$$
d\left(r\left(x_{1}\right), r\left(x_{2}\right)\right) \leq d\left(x_{1}, X\right)+d\left(x_{1}, x_{2}\right)+d\left(x_{2}, X\right) .
$$

For every $\epsilon>0$ there are only finitely many points $x \in X^{i}$ with $d(x, X) \geq \epsilon$, so continuity of $r$ at the points of $X$ follows. Continuity at the isolated points is trivial.

Notice that if $N_{0}$ is a cofinite subset of $X^{i} \backslash X$, then the closure of $N_{0}$ contains $X$, so $r\left(N_{0}\right)$ is dense in $X$.

We now recall the elegant proof of Lorch's Uniqueness Theorem, [18] Proposition 10.

Theorem 3.3. Every space $X$ has an essentially unique isolated point extension. That is, if $(Y, X)$ and $\left(Y^{\prime}, X\right)$ are point extension pairs, then there is a homeomorphism $f:(Y, X) \rightarrow\left(Y^{\prime}, X\right)$ rel $X$.

Proof: Let $\left\{x_{n}: n \in \mathbb{N}\right\}$ be a sequence of not necessarily distinct points in $X$ such that $\left\{x_{n}: n \geq N\right\}$ is dense in $X$ for all $N \in \mathbb{N}$. Let $X^{i}=X \times\{0\} \cup\left\{\left(x_{n}, n^{-1}\right): n \in \mathbb{N}\right\}$, and identify $X$ with $X \times\{0\}$. Clearly, $\left(X^{i}, X\right)$ is a point extension pair, so $X$ has at least one point extension.

Let $(Y, X)$ and $\left(Y^{\prime}, X\right)$ be point extension pairs.

Fix metrics $d$ and $d^{\prime}$ on $Y$ and $Y^{\prime}$, respectively. Define a metric $d^{\prime \prime}$ on $X$ as the pointwise maximum of $d$ and $d^{\prime}$. The three metrics $d, d^{\prime}$ and $d^{\prime \prime}$ are uniformly equivalent on $X$. Let $r: Y \rightarrow X$ and $r^{\prime}: Y^{\prime} \rightarrow X$ be canonical retractions. Let $N=\operatorname{Iso}(Y)=Y \backslash X$ and $N^{\prime}=\operatorname{Iso}\left(Y^{\prime}\right)=Y^{\prime} \backslash X$. Use a counting of $N$ and $N^{\prime}$ to impose orderings which are order isomorphic to $\mathbb{N}$ and so are well-orderings.

Let $a_{1}$ be the first element of $N$, and choose $b_{1}$ to be the first element of $N^{\prime}$ which satisfies

$$
d^{\prime \prime}\left(r\left(a_{1}\right), r^{\prime}\left(b_{1}\right)\right)<d\left(a_{1}, X\right) .
$$

Let $b_{2}$ be the first element of $N^{\prime} \backslash\left\{b_{1}\right\}$, and choose $a_{2}$ to be the first element of $N \backslash\left\{a_{1}\right\}$ such that

$$
d^{\prime \prime}\left(r\left(a_{2}\right), r^{\prime}\left(b_{2}\right)\right)<d^{\prime}\left(b_{2}, X\right) .
$$

Proceed inductively. If $n$ is even, let $a_{n+1}$ be the first element of $N \backslash\left\{a_{1}, \ldots, a_{n}\right\}$, and if $n$ is odd, let $b_{n+1}$ be the first element of $N^{\prime} \backslash$ $\left\{b_{1}, \ldots, b_{n}\right\}$. We can then choose $b_{n+1}$ or $a_{n+1}$ so that

$$
d^{\prime \prime}\left(r\left(a_{n+1}\right), r^{\prime}\left(b_{n+1}\right)\right)<\max \left\{d\left(a_{n+1}, X\right), d^{\prime}\left(b_{n+1}, X\right)\right\} .
$$

By construction, $\left\{a_{n}\right\}$ and $\left\{b_{n}\right\}$ are re-numberings of the sets $N$ and $N^{\prime}$. Define the mapping $f: Y \rightarrow Y^{\prime}$ as an extension of the identity on $X$ by putting $f\left(a_{n}\right)=b_{n}$ for all $n$. Let $m_{n}=\max \left\{d\left(a_{n}, X\right), d^{\prime}\left(b_{n}, X\right)\right\}$. Observe that $m_{n} \longrightarrow 0$ as $n \longrightarrow \infty$. 
Continuity of $f$ is clear at the isolated points. Suppose $x \in X$ and $a_{n_{i}} \longrightarrow x$ so that $n_{i} \longrightarrow \infty$.

$$
\begin{aligned}
d\left(r\left(a_{n_{i}}\right), x\right) & \leq d\left(a_{n_{i}}, x\right)+d\left(r\left(a_{n_{i}}\right), a_{n_{i}}\right) \\
& \leq d\left(a_{n_{i}}, x\right)+m_{n_{i}} \longrightarrow 0,
\end{aligned}
$$

and so $d^{\prime}\left(r\left(a_{n_{i}}\right), x\right) \longrightarrow 0$. Hence,

$$
\begin{aligned}
d^{\prime}\left(b_{n_{i}}, x\right) & \leq d^{\prime}\left(r\left(b_{n_{i}}\right), b_{n_{i}}\right)+d^{\prime}\left(r\left(b_{n_{i}}\right), r\left(a_{n_{i}}\right)\right)+d^{\prime}\left(r\left(a_{n_{i}}\right), x\right) \\
& \leq 2 m_{n_{i}}+d^{\prime}\left(r\left(a_{n_{i}}\right), x\right) \longrightarrow 0 .
\end{aligned}
$$

Continuity of $f$ follows. The result for $f^{-1}$ is similar and also follows from compactness.

Corollary 3.4. Let $\left(X^{i}, X\right)$ and $\left(Y^{i}, Y\right)$ be point extension pairs and $h: X \rightarrow Y$ a surjective continuous map. There exist a continuous map $H:\left(X^{i}, X\right) \rightarrow\left(Y^{i}, Y\right)$ which restricts to $h$ on $X$ and to a homeomorphism of $\operatorname{Iso}\left(X^{i}\right)=X^{i} \backslash X$ onto $\operatorname{Iso}\left(Y^{i}\right)=Y^{i} \backslash Y$. In particular, if $h$ is a homeomorphism, so is $H$.

Proof: We attach $Y$ to $X^{i}$ using $h$, letting $q_{h}: X^{i} \rightarrow X^{i} / h$ be the quotient map. We regard $Y$ as a subset of $X^{i} / h$ so that $\left(X^{i} / h, Y\right)$ is a point extension pair and $q_{h}: X^{i} \rightarrow X^{i} / h$ is an extension of $h$ which is a homeomorphism from $X^{i} \backslash X$ onto $X^{i} / h \backslash Y$. By Theorem 3.3 there is a homeomorphism $f:\left(X^{i} / h, Y\right) \rightarrow\left(Y^{i}, Y\right)$ rel $Y$. Let $H=f \circ q_{h}$.

Lemma 3.5. Let $\left(X_{1}^{i}, X_{1}\right)$ and $\left(X_{2}^{i}, X_{2}\right)$ be point extension pairs, and let $H:\left(X_{1}^{i}, X_{1}\right) \rightarrow\left(X_{2}^{i}, X_{2}\right)$ be a continuous map of pairs. Let $\left(Y_{1}, X_{1}\right)$ and $\left(Y_{2}, X_{2}\right)$ be extension pairs with $\pi_{1}:\left(Y_{1}, X_{1}\right) \rightarrow\left(X_{1}^{i}, X_{1}\right)$ and $\pi_{2}:\left(Y_{2}, X_{2}\right) \rightarrow\left(X_{2}^{i}, X_{2}\right)$ pair maps rel $X_{1}$ and rel $X_{2}$, respectively. Assume that $\widetilde{H}: Y_{1} \rightarrow Y_{2}$ is a function such that $\pi_{2} \circ \widetilde{H}=H \circ \pi_{1}$, i.e. the following diagram commutes:

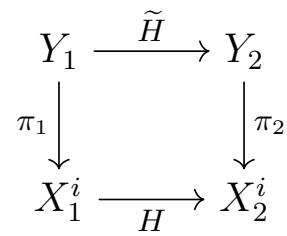

If for each $x \in \operatorname{Iso}\left(X_{1}^{i}\right)$ the restriction $\widetilde{H}: \pi_{1}^{-1}(x) \rightarrow \pi_{2}^{-1}(H(x))$ is continuous, then $\widetilde{H}$ is continuous on $Y_{1}$.

Proof: If $x \in \operatorname{Iso}\left(X_{1}^{i}\right)$, then $\pi_{1}^{-1}(x)$ is a clopen set, and so $\widetilde{H}$ is continuous at the points of $\pi_{1}^{-1}\left(\operatorname{Iso}\left(X^{i}\right)\right)$ by hypothesis.

Let $x \in X_{1}$, so $y=H(x) \in X_{2}$. Given $\epsilon>0$, there exists by (3.1) $\delta>0$ so that $\pi_{2}^{-1}\left(V_{\delta}^{d_{2}}(y)\right) \subset V_{\epsilon}^{d_{2}^{\prime}}(y)$, where $d_{2}$ and $d_{2}^{\prime}$ are the 
metrics on $X_{2}^{i}$ and $Y_{2}$, respectively. By continuity of $H$ there exists $\gamma>0$ so that $H\left(V_{\gamma}^{d_{1}}(x)\right) \subset V_{\delta}^{d_{2}}(y)$. If $x_{1} \in \pi_{1}^{-1}\left(V_{\gamma}^{d_{1}}(x)\right)$, then $\widetilde{H}\left(x_{1}\right) \in$ $\pi_{2}^{-1}\left(V_{\delta}^{d_{2}}(y)\right) \subset V_{\epsilon}^{d_{2}^{\prime}}(y)$. Hence, $\pi_{1}^{-1}\left(V_{\gamma}^{d_{1}}(x)\right)$ is a neighborhood of $x$ in $Y_{1}$ which is mapped by $\widetilde{H}$ into the neighborhood $V_{\epsilon}^{d_{2}^{\prime}}(y)$ in $Y_{2}$, and continuity at $x$ follows.

Definition 3.6. For a space $K$ and a pair of spaces $(Y, X)$, we call $Y$ a $K$-extension of $X$ if there exist a point extension pair $\left(X^{i}, X\right)$ and a map of pairs $\pi:(Y, X) \rightarrow\left(X^{i}, X\right)$ rel $X$ such that $\pi^{-1}(x)$ is homeomorphic to $K$ for every $x \in \operatorname{Iso}\left(X^{i}\right)$. We then call $(Y, X)$ a $K$-extension pair, and the space $Y$ is denoted by $X^{(K)}$.

We extend Lorch's Theorem.

Theorem 3.7. For any given space $K$, every space $X$ has an essentially unique $K$-extension pair $\left(X^{(K)}, X\right)$. Furthermore, if $\left(X^{(K)}, X\right)$ and $\left(Y^{(K)}, Y\right)$ are $K$-extension pairs and $h: X \rightarrow Y$ is a surjective continuous map, then there exists a continuous map $H:\left(X^{(K)}, X\right) \rightarrow$ $\left(Y^{(K)}, Y\right)$ which restricts to $h$ on $X$ and to a homeomorphism of $X^{(K)} \backslash$ $X$ onto $Y^{(K)} \backslash Y$. In particular, if $h$ is a homeomorphism, then so is $H$.

Proof: Let $\left(X^{i}, X\right)$ be a point extension of $X$. Let $\pi:\left(X^{i} \times K, X \times\right.$ $K) \rightarrow\left(X^{i}, X\right)$ be the map of pairs given by the first coordinate projection. Attach $X^{i} \times K$ to $X$ by using $h=\left.\pi\right|_{X \times K}$. The map $\pi$ factors through the quotient map $q_{h}$ to define a map $\left(\left(X^{i} \times K\right) / h, X\right) \rightarrow$ $\left(X^{i}, X\right)$ rel $X$. Thus, $\left(\left(X^{i} \times K\right) / h, X\right)$ is a $K$-extension pair.

Now let $\pi_{X}:\left(X^{(K)}, X\right) \rightarrow\left(X^{i}, X\right)$ and $\pi_{Y}:\left(Y^{(K)}, Y\right) \rightarrow\left(Y^{i}, Y\right)$ be maps rel $X$ and $Y$, respectively, with each fiber over $X^{i} \backslash X$ and $Y^{i} \backslash Y$ homeomorphic to $K$. Use Corollary 3.4 to get an extension $H^{i}:\left(X^{i}, X\right) \rightarrow\left(Y^{i}, Y\right)$ which maps $X^{i} \backslash X$ to $Y^{i} \backslash Y$ homeomorphically. Define $H$ by choosing for each $x \in X^{i} \backslash X$ an arbitrary homeomorphism from $\pi_{X}^{-1}(x)$ to $\pi_{Y}^{-1}\left(H^{i}(x)\right)$. These exist because each fiber is homeomorphic to $K$. By Lemma 3.5, the resulting $H$ is continuous.

In particular, if $Y=X$ and $h=1_{X}$, then it follows that the $K$ extension is essentially unique.

Two cases are of special interest to us. Recall that a component of a space $X$ is an isolated component if it is a clopen subset of $X$.

Theorem 3.8. Let $(Y, X)$ be an extension pair.

(a) If $K$ is a Cantor set, then $(Y, X)$ is a $K$-extension pair iff $Y$ is perfect and the dense, open set $Y \backslash X$ is zero-dimensional.

(b) If $K$ is a connected space, then $(Y, X)$ is a $K$-extension pair iff 
CHAIN TRANSITIVE HOMEOMORPHISMS ON A SPACE: ALL OR NONE 21

- The union of the isolated components is dense in $Y$.

- Each isolated component is homeomorphic to K.

- (diameter condition) For every $\epsilon>0$ there are only finitely many isolated components with diameter greater than $\epsilon$.

Proof: Let $\left\{A_{n}\right\}$ be a pairwise disjoint sequence of nonempty clopen subsets of $Y$ with union $Y \backslash X$. If for every $\epsilon>0$ only finitely many of the sets $A_{n}$ have diameter greater than $\epsilon$, then

$$
E=1_{X} \cup \bigcup_{n}\left\{A_{n} \times A_{n}\right\}
$$

is a closed equivalence relation. If $q: Y \rightarrow Y / E$ is the quotient space projection, then $(Y / E, X)$ is a point extension pair, $q$ is a map of pairs rel $X$, and the fibers of $q$ over the isolated points are the sets $A_{n}$. Conversely, if $\pi:(Y, X) \rightarrow\left(X^{i}, X\right)$ is a map of pairs rel $X$, then by (3.1) there are only finitely many fibers $\pi^{-1}(x)$ with diameter at least $\epsilon$.

(a) If $\pi:(Y, X) \rightarrow\left(X^{i}, X\right)$ is a map rel $X$ with each fiber over a point of $X^{i} \backslash X$ a Cantor set, then as a countable disjoint union of Cantor sets, $Y \backslash X$ is zero-dimensional and $\operatorname{Iso}(Y)=\emptyset$.

Conversely, if the locally compact space $Y \backslash X$ is zero-dimensional with no isolated points, then we can express it as the union of a pairwise disjoint sequence $\left\{C_{n}: n \in \mathbb{N}\right\}$ of nonempty, clopen subsets of $Y$ each of which is thus a Cantor set. Let $\left\{C_{n, i}: i=1, \ldots, N_{n}\right\}$ be a partition of $C_{n}$ by nonempty clopen subsets of diameter less than $n^{-1}$. If $\left\{A_{n}\right\}$ is a counting of the collection $\left\{C_{n, i}: n \in \mathbb{N}, i=1, \ldots, N_{n}\right\}$, then with $E$ as in (3.9) the projection $q:(Y, X) \rightarrow(Y / E, X)$ is a map rel $X$ with each fiber over a point of $(Y / E) \backslash X$ a Cantor set. Thus, $(Y, X)$ is a Cantor set extension pair.

(b) If $\pi:(Y, X) \rightarrow\left(X^{i}, X\right)$ is a map rel $X$ with each fiber connected, then $\left\{\pi^{-1}(x): x \in X^{i} \backslash X\right\}$ is the set of isolated components, so the conditions of (b) are necessary.

Conversely, if they hold, then we let $\left\{A_{n}\right\}$ be the sequence of isolated components. There are infinitely many isolated components because $X$ is nonempty and $Y \backslash X$ is dense. With $E$ as in (3.9) again, $q:(Y, X) \rightarrow$ $(Y / E, X)$ is the required map rel $X$.

Corollary 3.9. If $Y$ and $X$ are Cantor sets with closed, nowhere dense subsets $Y_{1} \subset Y$ and $X_{1} \subset X$, then for any surjective continuous map $h: Y_{1} \rightarrow X_{1}$ there is a continuous map $H: Y \rightarrow X$ which extends $h$ and which restricts to a homeomorphism of $Y \backslash Y_{1}$ to $X \backslash X_{1}$. In particular, if $h$ is a homeomorphism, then so is $H$. 
Proof: By Theorem 3.8 (a), $\left(Y, Y_{1}\right)$ and $\left(X, X_{1}\right)$ are Cantor set extension pairs. The existence of $H$ then follows from Theorem 3.7.

Remark: This is a classical theorem of Knaster and Reichbach, extended by Gutek, see [17] and [14].

Now we apply these results.

Lemma 3.10. Let $\left(X^{i}, X\right)$ be a point extension pair, $(Y, X)$ an extension pair and $\pi:(Y, X) \rightarrow\left(X^{i}, X\right)$ a surjective map of pairs rel $X$.

(a) The map $\pi: Y \rightarrow X^{i}$ is open.

(b) Assume that $H:(Y, X) \rightarrow(Y, X)$ and $H^{i}:\left(X^{i}, X\right) \rightarrow\left(X^{i}, X\right)$ are homeomorphisms with $\pi$ mapping $H$ to $H^{i}$, i.e. $\pi \circ H=$ $H^{i} \circ \pi$. If $H^{i}$ is chain transitive, then $H$ is chain transitive.

Proof: (a) If $U \subset X$ is open and $x \in \pi(U) \cap\left(X^{i} \backslash X\right)$, then $\pi(U)$ is a neighborhood of $x$ because $x$ is an isolated point.

If $x \in \pi(U) \cap X$, then there exist $\epsilon>0$ and $\delta>0$ so that $\bar{V}_{\epsilon}^{d_{Y}}(x) \subset$ $U$ and $\pi^{-1}\left(\bar{V}_{\delta}^{d_{X^{i}}}(x)\right) \subset \bar{V}_{\epsilon}^{d_{Y}}(x)$. Since $\pi$ is surjective, $\pi\left(\bar{V}_{\epsilon}^{d_{Y}}(x)\right) \supset$ $\bar{V}_{\delta}^{d_{X^{i}}}(x)$, so $\pi(U)$ is a neighborhood of $x$.

(b) Since $\pi$ is open, Proposition 2.4 (e) implies that $\pi$ is a semiconjugacy from $\mathrm{e} H$ to $\mathcal{C} H^{i}$ and from $\mathcal{C} H^{-1}$ to $\mathcal{C}\left(H^{i}\right)^{-1}$. Fix $x \in X$. For any $y \in Y$ we have $x \in \mathcal{C} H^{i}(\pi(y))$ and $x \in \mathcal{C}\left(H^{i}\right)^{-1}(\pi(y))$ because $H^{i}$ is chain transitive. Since $\{x\}=\pi^{-1}(x)$, it follows from the semiconjugacy that $x \in \mathcal{C} H(y)$ and $x \in \mathcal{C} H^{-1}(y)$. That is, every point of $Y$ is chain equivalent to $x$, and so transitivity of $\mathcal{C} H$ implies that $H$ is chain transitive.

Theorem 3.11. Let $\left(X^{i}, X\right)$ be a point extension pair, $\left(X^{c}, X\right)$ a Cantor set extension pair and $\left(X^{(K)}, X\right)$ a $K$-extension pair for spaces $X$ and $K$.

(a) If $f$ is a chain transitive homeomorphism on $X$, then there exists a homeomorphism $F$ on $X^{i}$ which extends $f$ on $X$ such that

- $F$ is chain transitive,

- $F_{ \pm}$is topologically transitive, and

- if $x \in X^{i} \backslash X$, then $\omega F(x)=X=\alpha F(x)$.

(b) There exists $G^{c}$ a topologically transitive homeomorphism on $X^{c}$ which extends $1_{X}$.

(c) There exists $G^{K}$ a chain transitive homeomorphism on $X^{(K)}$ which extends $1_{X}$.

Proof: (a) By concatenating $\epsilon$-chains which are $\epsilon$-dense in $X$, we can obtain an infinite sequence $\left\{x_{k}: k \in \mathbb{Z}\right\}$ with $d\left(f\left(x_{k}\right), x_{k+1}\right) \longrightarrow 0$ as 
$|k| \longrightarrow \infty$ and so that for any $N \in \mathbb{N}$ the tails $\left\{x_{k}: k \geq N\right\}$ and $\left\{x_{-k}:\right.$ $k \geq N\}$ are dense in $X$. Define the sequence $\left\{y_{k} \in X \times[0,1]: k \in \mathbb{Z}\right\}$ by

$$
y_{k}= \begin{cases}\left(x_{k},(2 k+1)^{-1}\right) & \text { for } k \geq 0 \\ \left(x_{k},(2|k|)^{-1}\right) & \text { for } k<0\end{cases}
$$

Let $Y=X \times\{0\} \cup\left\{y_{k}: k \in \mathbb{Z}\right\}$, and define $\bar{F}(x, 0)=(f(x), 0)$ and $\bar{F}\left(y_{k}\right)=y_{k+1}$ for $k \in \mathbb{Z}$. It is easy to see that $Y$ is an isolated point extension of $X=X \times\{0\}, \bar{F}$ is a homeomorphism on $Y$, and $X \times\{0\}=$ $\omega \bar{F}\left(y_{k}\right)=\alpha \bar{F}\left(y_{k}\right)$ for any $k \in \mathbb{Z}$. Since the orbit $\mathcal{O}\left(\bar{F}_{ \pm}\right)\left(y_{0}\right)=\left\{y_{k}:\right.$ $k \in \mathbb{Z}\}$ is dense, it follows that $\bar{F}_{ \pm}$is topologically transitive. The homeomorphism $\bar{F}$ is chain transitive on $Y$ because $f$ is chain transitive on $X$ and because $X=\omega \bar{F}\left(y_{k}\right) \subset \mathcal{e} \bar{F}\left(y_{k}\right)$ and also $X=\alpha \bar{F}\left(y_{k}\right) \subset$ $\mathrm{e} \bar{F}^{-1}\left(y_{k}\right)$.

By Lorch's Uniqueness Theorem 3.3 there exists a homeomorphism $H:\left(X^{i}, X\right) \rightarrow(Y, X)$ rel $X$. Let $F=H^{-1} \circ \bar{F} \circ H$.

(b) We begin with $G$ a topologically transitive homeomorphism on a Cantor set with a Cantor set $C$ of fixed points. By topological transitivity, $C$ is necessarily nowhere dense. To be specific, let $G$ be the shift homeomorphism on the product space $C^{\mathbb{Z}}$. Let $c: C \rightarrow C^{\mathbb{Z}}$ be the embedding with $c(x)_{i}=x$ for all $i \in \mathbb{Z}$. Thus, $c$ is a homeomorphism onto the set of fixed points. Since $C$ is nowhere dense, $\left(C^{\mathbb{Z}}, C\right)$ is a Cantor set extension pair by Theorem 3.8 (a).

For an arbitrary space $X$, there exists by Proposition 2.9 a continuous surjection $h: C \rightarrow X$. We attach $C^{\mathbb{Z}}$ to $X$ using $h$. Let $Y=\left(C^{\mathbb{Z}}\right) / h$. Now, $(Y, X)$ is a Cantor set extension pair, and $G$ factors to define a topologically transitive homeomorphism $\bar{G}$ which restricts to the identity on $X$.

By Theorem 3.7 there exists a homeomorphism $H:\left(X^{c}, X\right) \rightarrow$ $(Y, X)$ rel $X$. Let $G^{c}=H^{-1} \circ \bar{G} \circ H$.

(c) First we consider the case where $X$ is the usual Cantor set $C$ in $[0,1]$ with $0,1 \in C$. Then the complement consists of a pairwise disjoint, countable collection $\left\{\left(a_{i}, b_{i}\right): i \in \mathbb{N}\right\}$ of open intervals in $(0,1)$. Let $\ell_{i}=b_{i}-a_{i}$, so $\ell_{i}>0$ for all $i \in \mathbb{N}$, but for any $\epsilon>0$ there are only finitely many with $\ell_{i} \geq \epsilon$. Let

$$
C^{i}=C \times\{0\} \cup \bigcup_{i, m \in \mathbb{N}}\left\{\left(a_{i}, \ell_{i} / m\right),\left(b_{i}, \ell_{i} / m\right)\right\}
$$

Since the set of endpoints is dense in $C$, it follows that $C^{i}$ is a point extension of $C=C \times\{0\}$. Now we relabel the isolated points. For 
$i \in \mathbb{N}, k \in \mathbb{Z}$, define

$$
\begin{aligned}
& u_{i, k}= \begin{cases}\left(a_{i}, \ell_{i} \cdot(2 k+1)^{-1}\right) & \text { for } k \geq 0 \\
\left(b_{i}, \ell_{i} \cdot(2|k|)^{-1}\right) & \text { for } k<0\end{cases} \\
& v_{i, k}= \begin{cases}\left(b_{i}, \ell_{i} \cdot(2 k+1)^{-1}\right) & \text { for } k \geq 0 \\
\left(a_{i}, \ell_{i} \cdot(2|k|)^{-1}\right) & \text { for } k<0\end{cases}
\end{aligned}
$$

Define $G$ as an extension of $1_{C}$ so that $u_{i, k} \stackrel{G}{\longmapsto} u_{i, k+1}$ and $v_{i, k} \stackrel{G}{\longmapsto} v_{i, k+1}$. Thus, above each endpoint $a_{i}$ the $u_{i, k}$ 's runs up the $\left(a_{i}, \ell_{i} / m\right)$ 's with $m$ even, jumps from $\left(a_{i}, \ell_{i} / 2\right)$ to $\left(b_{i}, \ell_{i}\right)$, and then moves down the $\left(b_{i}, \ell_{i} / m\right)$ 's with $m$ odd. The $v_{i, k}$ 's provide a similar path from $b_{i}$ to $a_{i}$. Since for any $\epsilon>0$ at most finitely many points move a distance more than $\epsilon$, it follows that $G$ and its inverse are continuous.

It is clear that for any $i$ the points of $\left\{\left(a_{i}, \ell_{i} / m\right),\left(b_{i}, \ell_{i} / m\right): m \in \mathbb{N}\right\}$ all lie in a single chain component. Given $\epsilon>0$, it is clear that we can get from a point $x \in C$ to a point $y \in C$ by an $\epsilon$-chain jumping across the gaps of length less than $\epsilon$ which occur between $x$ and $y$. For the finite number of remaining gaps we use the isolated point orbits to get across. Hence, $G$ is chain transitive on $C^{i}$.

For an arbitrary space $X$, we again use a continuous surjection $h$ : $C \rightarrow X$ from Proposition 2.9. Let $X^{i}$ be the quotient space $C^{i} / h$ obtained by attaching $X$ via $h$. Then, $G \cup 1_{X}$ on $C^{i} \cup X$ factors through the quotient map $q_{h}$ to define a homeomorphism $G^{i}$. Because $q_{h}$ maps $G$ on $C^{i}$ onto $G^{i}$ on $X^{i}$ it follows that $G^{i}$ is chain transitive by Proposition 2.4(d). Because $q_{h}: C^{i} \backslash C \rightarrow X^{i} \backslash X$ is a homeomorphism, it follows that $X^{i}$ is an isolated point extension of $X$.

Because $\left(X^{(K)}, X\right)$ is a $K$-extension pair and the point extension is essentially unique, there exists $\pi:\left(X^{(K)}, X\right) \rightarrow\left(X^{i}, X\right)$, a map of pairs rel $X$, such that the fiber $\pi^{-1}(x)$ is homeomorphic to $K$ for every $x \in X^{i} \backslash X$. For each such $x$ let $G^{K}$ restrict to a homeomorphism from $\pi^{-1}(x)$ to $\pi^{-1}\left(G^{i}(x)\right)$. By Lemma 3.5 $G^{K}$ and its inverse are continuous. By Lemma 3.10 $G^{K}$ is chain transitive because $G^{i}$ is.

Remark: By Proposition 2.5 (b) there is no topologically transitive homeomorphism on a space with infinitely many isolated points. Hence, the result in (a) above is the best we can hope for. In particular, we see that any chain transitive homeomorphism on $X$ can be extended to a system in which $X$ is an omega limit set.

Recall from Proposition 2.7 (e) that if $X$ is connected, then $1_{X}$ is chain transitive.

Now we can prove a slight extension of Theorem 1.1. 
Corollary 3.12. For a space $X$ let $X_{1}$ be the closure of the union of all components which meet $\overline{\operatorname{Iso}(X)}$. If $X_{1}$ is a proper, clopen, nonempty subset of $X$, then $X$ is $H(X)$-decomposable and so $X$ admits no chain transitive homeomorphism. If $X_{1}$ is not a proper clopen subset of $X$ and the open set $X \backslash X_{1}$ is empty or zero-dimensional, then $X$ admits a chain transitive homeomorphism.

Proof: The sets $\overline{\operatorname{Iso}(X)}$ and $X_{1}$ are $h_{X}$-invariant, so if $X_{1}$ is proper, clopen and nonempty, then $X$ is $h_{X}$-decomposable.

Assume that $X_{1}$ is not a proper clopen subset of $X$. If $\operatorname{Iso}(X)$ is finite and nonempty, then $X=X_{1}=\operatorname{Iso}(X)$ and we can define $f$ so that $X$ consists of a single periodic orbit. If $\operatorname{Iso}(X)=\emptyset$, then $X_{1}=\emptyset$ and $X=X \backslash X_{1}$ is zero-dimensional and perfect, and so $X$ is a Cantor set. Hence, $X$ admits a topologically transitive homeomorphism.

Now assume that $\operatorname{Iso}(X)$ is infinite, so $A=\overline{\operatorname{Iso}(X)} \backslash \operatorname{Iso}(X)$ is nonempty. Clearly, $(\overline{\operatorname{Iso}(X)}, A)$ is a point extension pair, and by Theorem 3.11(c) there exists a chain transitive homeomorphism $f_{1}$ on $\overline{\operatorname{Iso}(X)}$ which is the identity on $A$. Extend $f_{1}$ to be the identity on $X_{1} \backslash \operatorname{Iso}(X)$. Thus, $f_{1}$ is the identity on every nontrivial component of $X$ which meets $\overline{\mathrm{Iso}}(X)$. It follows from Proposition 2.7 (e) that all of these are contained in the chain component of $f_{1}$ which contains all of $\overline{\operatorname{Iso}(X)}$. As this chain component is closed, it must contain all of $X_{1}$. That is, $f_{1}$ on $X_{1}$ is chain transitive. If $X=X_{1}$, then we are done.

Otherwise, the nonempty, open, zero-dimensional set $X \backslash X_{1}$ is not closed and contains no isolated points. So $X_{2}=\overline{X \backslash X_{1}}$ is perfect and $B=X_{2} \cap X_{1}$ is nonempty subset of $X_{1}$ disjoint from $\operatorname{Iso}(X)$. We see that $\left(X_{2}, B\right)$ is a Cantor set extension pair, so by Theorem 3.11(b) there exists a topologically transitive homeomorphism $f_{2}$ on $X_{2}$ which restricts to the identity on $B$.

The concatenation $f=f_{1} \cup f_{2}$ is a homeomorphism on $X$. Since $f_{1}$ and $f_{2}$ are chain transitive and $X_{1} \cap X_{2} \neq \emptyset$ it follows that all of $X$ is contained in a single chain component, i.e. $f$ is chain transitive.

To extend these results we need some simple lifting facts.

Lemma 3.13. Let $f_{i} \in H\left(X_{i}\right)$ for $i=1,2$ and let $\pi: X_{1} \rightarrow X_{2}$ be a continuous surjection mapping $f_{1}$ to $f_{2}$. Assume that $(\pi \times \pi)^{-1}\left(1_{X_{2}}\right) \subset$ $\mathcal{e} f_{1}$. That is, each fiber of $\pi$ is entirely contained in a single chain component of $f_{1}$.

(a) Both $f_{1}$ and $f_{2}$ are chain recurrent, i.e.

$$
1_{X_{1}} \subset \mathcal{e} f_{1} \text { and } 1_{X_{2}} \subset \mathcal{e} f_{2} .
$$

(b) The space $X_{1}$ is $f_{1}$-decomposable iff $X_{2}$ is $f_{2}$-decomposable. 
(c) The chain relations satisfy

$$
\mathcal{C} f_{2}=(\pi \times \pi)\left(\bigodot f_{1}\right) \text { and } \mathcal{C} f_{1}=(\pi \times \pi)^{-1}\left(\bigodot f_{2}\right) .
$$

(d) The homeomorphism $f_{1}$ is chain transitive iff $f_{2}$ is chain transitive.

Proof: (a) Let $E_{\pi}=(\pi \times \pi)^{-1}\left(1_{X_{2}}\right)$. It is a closed equivalence relation and so contains $1_{X_{1}}$. Hence, $1_{X_{1}} \subset E_{\pi} \subset \mathcal{C} f_{1}$.

Because $\pi$ is surjective, $1_{X_{2}}=(\pi \times \pi)\left(1_{X_{1}}\right)$. Since $\pi$ maps $f_{1}$ to $f_{2}$, it maps $\mathcal{e} f_{1}$ to $\mathcal{C} f_{2}$ by Proposition 2.4(d). Hence,

$$
1_{X_{2}}=(\pi \times \pi)\left(1_{X_{1}}\right) \subset(\pi \times \pi)\left(\mathcal{C} f_{1}\right) \subset \mathcal{C} f_{2} .
$$

(b) If $B$ and its complement are proper, clopen, forward $f_{2}$-invariant subsets of $X_{2}$ then because $\pi$ is surjective, $\pi^{-1}(B)$ and its complement are proper, clopen, forward $f_{1}$-invariant subsets of $X_{1}$.

Now assume that $A$ and its complement are proper, clopen, forward $f_{1}$-invariant subsets of $X_{1}$. Since each is forward $\mathcal{C} f_{1}$-invariant, it follows that each is saturated by the equivalence relation $E_{\pi} \subset \mathcal{e} f_{1}$. Hence, $\pi(A)$ and $\pi\left(X_{1} \backslash A\right)$ are disjoint closed sets with union $\pi\left(X_{1}\right)=$ $X_{2}$. That is, they are complementary clopen sets. Furthermore, they are forward $f_{2}$-invariant.

(c) As mentioned above, $(\pi \times \pi)\left(\mathcal{C} f_{1}\right) \subset \mathcal{e} f_{2}$. Now assume $\left(x_{1}, x_{2}\right) \notin$ $\mathcal{e} f_{1}$. Since $X_{1}=\left|\mathcal{e} f_{1}\right|$ by $(\mathrm{a})$, Proposition 2.1(c) implies there is an attractor $A$ for $f_{1}$ which contains $x_{1}$ but not $x_{2}$, and by Proposition 2.1(d) $A$ is a clopen $\mathcal{C} f_{1}$-invariant set. Hence, it is saturated by $E_{\pi}$. So $\pi(A)$ is clopen and $f_{2}$-invariant, and therefore also $\mathcal{C} f_{2}$-invariant. Now, $\pi\left(x_{1}\right) \in \pi(A)$, and $x_{2} \notin A=\pi^{-1}(\pi(A))$ implies $\pi\left(x_{2}\right) \notin \pi(A)$. It follows that $\left(\pi\left(x_{1}\right), \pi\left(x_{2}\right)\right) \notin \mathcal{C} f_{2}$. Thus, the complement of $\mathcal{C} f_{1}$ in $X_{1} \times X_{1}$ maps into the complement of $\mathcal{e} f_{2}$. Since $\pi \times \pi$ is surjective, the equations of (c) follow.

(d) Immediate from (c) and the surjectivity of $\pi$.

For any space $X$, the chain relation $\mathcal{C} 1_{X}$ is a closed equivalence relation with equivalence classes the components of $X$. Let $[X]$ be the zero-dimensional space of components, the quotient space for this equivalence relation with quotient map $\pi_{X}: X \rightarrow[X]$, see Lemma 2.6(b) and Proposition [2.1(d). There is a natural homomorphism [.] : $H(X) \rightarrow H([X])$ with $\pi_{X}$ mapping $f$ to $[f]$ for $f \in H(X)$. Thus, $H(X)$ acts on $[X]$ and we let $\left[h_{X}\right]=\bigcup\{[h]: h \in H(X)\}$ be the associated relation on $[X]$.

\section{Proposition 3.14.}

(a) A space $X$ is $h_{X}$-decomposable iff $[X]$ is $\left[h_{X}\right]$-decomposable. 
(b) If $f$ is a chain recurrent homeomorphism on a space $X$, then $\left(\pi_{X} \times \pi_{X}\right)^{-1}\left(1_{[X]}\right) \subset \mathcal{C} f$. In that case, the following are equivalent:

(i) The map $f$ is chain transitive.

(ii) The space $X$ is $f$-indecomposable.

(iii) The map $[f]$ is chain transitive.

(iv) The space $[X]$ is $[f]$-indecomposable.

Proof: (a) This is clear because any clopen set is saturated by the equivalence relation $\mathrm{C} 1_{X}$.

(b) Since the space of chain components is zero-dimensional, every connected set of chain recurrent points is contained in a single chain component. If $f$ is chain recurrent, then every point is chain recurrent, so each component is contained in a single chain component. It follows that $\left(\pi_{X} \times \pi_{X}\right)^{-1}\left(1_{[X]}\right) \subset \mathcal{C} f$. Since $f$ and $[f]$ are chain recurrent, the equivalences (i) $\Leftrightarrow$ (ii) and (iii) $\Leftrightarrow$ (iv) follow from Proposition 2.7 (d). The implication (i) $\Rightarrow$ (iii) holds because $\pi$ maps $f$ to $[f]$. The converse (iii) $\Rightarrow$ (i) follows from Theorem 3.13 .

Thus, a chain transitive homeomorphism on $[X]$ lifts to a chain transitive homeomorphism iff it lifts to a chain recurrent homeomorphism.

Recall that a component $K$ of $X$ is an isolated component if it is a clopen subset of $X$.

For a space $X$ let $\mathcal{J}_{X}$ denote the set of isolated components. Two isolated components $K_{1}$ and $K_{2}$ are $H(X)$-equivalent if they are homeomorphic or, equivalently, if there exists $g \in H(X)$ such that $g\left(K_{1}\right)=$ $K_{2}$. Let $I_{X}$ be the set of $H(X)$-equivalence classes in $\mathcal{J}_{X}$. For $i \in I_{X}$ let $Q_{i}$ be the union of the isolated components in $i$, and let $Q$ be the union of all of the isolated components, so that $Q$ is the disjoint union of the $Q_{i}$ 's. Thus, $Q$ and each of the $Q_{i}$ 's are open subsets of $X$.

Lemma 3.15. If $A$ is a clopen $H(X)$-invariant set which meets some $\overline{Q_{i}}$, then it contains $\overline{Q_{i}}$. In particular, if all the isolated components are homeomorphic to one another and the union of the isolated components is dense, then $X$ is $H(X)$-indecomposable.

Proof: Since $A$ is open, it meets some isolated component $K \in i$. Since it is clopen, it contains $K$. If $K_{1} \in Q_{i}$, then there exists $g \in H(X)$ such that $g(K)=K_{1}$, and so $H(X)$-invariance implies $K_{1} \subset A$. Since $Q_{i} \subset A$ and $A$ is closed, we get $\overline{Q_{i}} \subset A$.

We will say that $X$ satisfies the diameter condition on isolated components if for every $\epsilon>0$ there are only finitely many isolated components with diameter greater than $\epsilon$.

The following is the furthest we can extend Corollary 3.12 , 
Theorem 3.16. For a space $X$, let $X_{1}$ be the closure of the union of all components which meet the closure of the union of all isolated components. Assume that $X$ satisfies the diameter condition on isolated components and that the open set $X \backslash X_{1}$ is empty or zero-dimensional.

(a) Either $X$ is $H(X)$-decomposable or $X$ admits a chain transitive homeomorphism.

(b) If $X_{1}$ is a proper clopen subset of $X$, then $X$ is $H(X)$-decomposable, and so $X$ admits no chain transitive homeomorphism.

(c) If $X_{1}$ is not a proper clopen subset of $X$ and all the isolated components are homeomorphic to one another, then $X$ admits a chain transitive homeomorphism.

Proof: (b) Obvious since $X_{1}$ is $H(X)$-invariant.

In (a) and (c) the extension from the closure of the isolated components to the rest of $X$ proceeds just as in Corollary 3.12 . So from now on we will assume that the union $Q$ of the isolated components is dense.

(c) If there are only $n$ isolated components, then their union $Q$ is clopen and so is all of $X$. By assumption they are all homeomorphic to some common space $K$. We can choose a homeomorphism with $f^{n}=$ $1_{X}$, and so that each periodic orbit meets each component. Clearly, $f$ is chain transitive.

Now we may assume that $\mathcal{J}_{X}$ is infinite, and let $A=X \backslash Q$. Thus, $A$ is a nonempty, closed nowhere dense set. By Theorem $3.8(\mathrm{~b})(X, A)$ is a $K$-extension pair. By Theorem 3.11 (c) $X$ admits a chain transitive homeomorphism rel $A$.

(a) We may assume that there is more than one equivalence class in $I_{X}$, for otherwise we are in case (c). If any $i \in I_{X}$ is finite, then $Q_{i}$ is a clopen $H(X)$-invariant set, so $X$ is $H(X)$-decomposable since $X \neq Q_{i}$ by the assumption that $I_{X}$ contains more than one class.

Now assume that every equivalence class in $I_{X}$ is infinite. Then the closure of each open $H(X)$-invariant set $Q_{i}$ meets $A$, and we let $A_{i}=\overline{Q_{i}} \cap A$. If $i \neq j$, then $\overline{Q_{i}} \cap \overline{Q_{j}} \subset A$, and so this intersection equals $A_{i} \cap A_{j}$.

Applying the argument for (c) to $\overline{Q_{i}}$, there exists a homeomorphism $f_{i}$ on $\overline{Q_{i}}$ which is chain transitive and which restricts to the identity on $A_{i}$.

Let $f$ on $X$ equal $f_{i}$ on $\overline{Q_{i}}$ and the identity on $A$. Because the diameter condition holds, we can apply Lemma 3.10 to see that $f$ is a homeomorphism on $X$. Since each $f_{i}$ is chain transitive, $\overline{\bigcup_{i}\left\{Q_{i} \times Q_{i}\right\}} \subset \mathcal{C} f$.

Let $x \in X$ and $g \in H(X)$. Since $Q=\bigcup_{i} Q_{i}$ is dense in $X$, there is a sequence $\left\{x_{k} \in Q_{i_{k}}\right\}$ which converges to $x$. Then $g\left(x_{k}\right) \in Q_{i_{k}}$, and so 
$(x, g(x)) \in \overline{\bigcup_{i} Q_{i} \times Q_{i}}$. Hence, $h_{X} \subset \mathcal{C} f$. In particular, $1_{X} \subset h_{X}$ implies that $f$ is chain recurrent. So by Proposition 2.7(d) $f$ is chain transitive iff $X$ is $f$-indecomposable. Since $h_{X} \subset \mathcal{e} f$, an $f$-decomposition, which is a $\mathcal{C} f$-decomposition, is also an $h_{X}$-decomposition. Hence, if $X$ is $f$-indecomposable, then it is $h_{X}$-indecomposable, i.e. $X$ is $H(X)$ indecomposable. On the other hand, if $X$ is $H(X)$-decomposable, then there does not exist any chain transitive homeomorphism on $X$.

As we will see below, the diameter condition on isolated components is essential for this result.

Example 3.17. We construct $X$ so that

- The connected components of $X$ are all homeomorphic to $[0,1]$.

- The space $X$ is $H(X)$-decomposable.

- There are no isolated components.

Let $C$ be a Cantor set and $S=\left\{a_{n}: n \in \mathbb{N}\right\}$ a sequence of distinct points in $C$ with closure $A$ in $C$. Define

$$
\begin{gathered}
I_{n}=\left\{\left(a_{n}, t\right): 0 \leq t \leq n^{-1}\right\} \subset C \times[0,1] \text { for } n \in \mathbb{N}, \\
C_{0}=C \times\{0\}, \quad C_{+}=C_{0} \cup \bigcup_{n} I_{n}, \\
C_{ \pm}=C_{+} \cup C \times[-1,0], \\
A_{0}=A \times\{0\}, \quad A_{+}=\longdiv { \bigcup _ { n } I _ { n } , } \\
A_{ \pm}=A_{+} \cup A \times[-1,0] .
\end{gathered}
$$

Each $I_{n}^{\circ}=I_{n} \backslash C \times\{0\}$ is open in $C_{ \pm}$, and so the points of each $I_{n}^{\circ}$ have connected neighborhoods. Hence, $A, A_{+}$and $A_{ \pm}$are $H\left(C_{ \pm}\right)$invariant. Thus, if $A$ is a proper clopen subset of $C$, then $C_{ \pm}$is $H\left(C_{ \pm}\right)$decomposable. Observe that every component is homeomorphic to the unit interval and the first coordinate projection maps $C_{ \pm}$onto the Cantor set $C$.

Also, if $A$ is a proper clopen set, then $C \times[-1,0]$ admits chain transitive homeomorphisms, but the factor $X=C \cup A \times[-1,0]$ is $H(X)$-decomposable.

A homeomorphism $f$ on $C$ can be extended to a homeomorphism $F_{+}$of $C_{+}$iff $A$ is $f$-invariant. In that case, we can then define $F_{+}$ by using any orientation-preserving homeomorphism from $I_{x}$ to $I_{f(x)}$, i.e. one which maps $(x, 0)$ to $(f(x), 0)$. Here $I_{x}=I_{n}$ for $x=a_{n}$ and $=\{(x, 0)\}$ if $x \notin S$. Continuity at points of $A_{+}^{\circ}$ is clear, and if $x \in C$, then for every $\epsilon$ there is a neighborhood $U$ of $x$ in $C$ so that $y \in U \backslash\{x\}$ implies that the length of the interval $I_{f(y)}$ is less than $\epsilon$. This implies 
continuity at $(x, 0)$. Notice that if $\mathcal{O}(x)$ is infinite, then

$$
\lim _{|n| \rightarrow \infty}\left|I_{f^{n}(x)}\right|=0,
$$

where $|J|$ denotes the length of an interval $J$. This says that any pair $\left(x, t_{1}\right),\left(x, t_{2}\right) \in I_{x}$ is asymptotic for $F_{+}$and $\left(F_{+}\right)^{-1}$ with

$$
\begin{aligned}
& \omega F_{+}\left(x, t_{1}\right)=\omega F_{+}\left(x, t_{2}\right)=\omega f(x) \times\{0\} \subset C_{0}, \\
& \alpha F_{+}\left(x, t_{1}\right)=\alpha F_{+}\left(x, t_{2}\right)=\alpha f(x) \times\{0\} \subset C_{0} .
\end{aligned}
$$

Now assume that $A$ is not clopen. If $f$ is chain transitive and every point of $A$ has an infinite orbit, then any extension $F_{+}$to $C_{+}$is chain transitive. Observe that $F_{+}$is chain transitive if, whenever $a_{n}$ is a periodic point for $f$, we define $F_{+}$by using the unique linear, orientationpreserving homeomorphism from $I_{a_{n}}$ to $I_{f\left(a_{n}\right)}$. On the other hand, if $f\left(a_{1}\right)=a_{1}$ and on $I_{a_{1}}$ we define $F_{+}$by $\left(a_{1}, t\right) \mapsto\left(a_{1}, t^{2}\right)$ then $\left(a_{1}, 1\right)$ is a repelling fixed point for $F_{+}$on $I_{a_{1}}$ and hence for $F_{+}$on $C_{+}$. If $F_{+}$is chain transitive, then we can obtain a chain transitive extension $F_{ \pm}$on $C_{ \pm}$by using $f \times 1_{[-1,0]}$ on $C \times[-1,0]$.

Example 3.18. We construct $X$ so that

- The connected components of $X$ are all homeomorphic to $[0,1]$.

- The space of connected components, $[X]$, consists of a convergent sequence and its limit, and the union of isolated components in $X$ is dense.

- It is $f$-decomposable for any $f \in H(X)$ but not $H(X)$-decomposable.

The space $X$ we construct is $H(X)$-indecomposable by the first two properties and by Lemma 3.15 .

For every $n \in \mathbb{N}$ we define $I_{n}=\left[0, n^{-1}\right]$ and a continuous function $t_{n}: I_{n} \rightarrow I=[0,1]$ so that, for integers $i=0, \ldots,(2 n)$ !,

$$
t_{n}\left(\frac{i}{n(2 n) !}\right)= \begin{cases}0 & \text { when } i \text { is even } \\ 1 & \text { when } i \text { is odd }\end{cases}
$$

and the rest of the values are defined by linear interpolations.

Each interval $I_{n}$ contains $(2 n)$ ! intervals $\left\{I_{n}^{i}: i=1, \ldots,(2 n) !\right\}$ of equal length, each of which is mapped by $t_{n}$ onto $I$. We can further subdivide each $I_{n}^{i}$ into intervals $\left\{I_{n}^{i, j}: j=1, \ldots, n\right\}$ of equal length so that each is mapped to a subinterval of $I$ of length $n^{-1}$ by $t_{n}$. The corresponding restrictions of $t_{n}$ are denoted by $t_{n}^{i}=\left.t_{n}\right|_{I_{n}^{i}}$ and $t_{n}^{i, j}=$ $\left.t_{n}\right|_{I_{n}^{i, j}}$ 
Regarding the functions $t_{n}, t_{n}^{i}$ and $t_{n}^{i, j}$ as closed subsets of $I_{n} \times I$, we define

$$
\begin{aligned}
X_{n} & =\left\{n^{-1}\right\} \times t_{n}, \quad n \in \mathbb{N}, \\
X_{\infty} & =\{(0,0)\} \times[0,1], \\
X & =\bigcup_{n} X_{n} \cup X_{\infty} .
\end{aligned}
$$

The space $X$ is clearly a closed, bounded subset of $\mathbb{R}^{3}$, and the space $[X]$ can be identified with $\pi(X)$, where $\pi: X \rightarrow\left\{n^{-1}: n \in \mathbb{N}\right\} \cup\{0\}$ is the projection to the first coordinate. The union of the isolated components is clearly dense in $X$. In addition, for all appropriate $n, i$ and $j$, we define

$$
X_{n}^{i}=\left\{n^{-1}\right\} \times t_{n}^{i} \quad \text { and } \quad X_{n}^{i, j}=\left\{n^{-1}\right\} \times t_{n}^{i, j} .
$$

Note that each $X_{n}$ is a union of the line segments $X_{n}^{i}$. Similarly, each $X_{n}^{i}$ is a union of the line segments $X_{n}^{i, j}$. The diameter of each $X_{n}^{i}$ is greater than 1, and the diameter of each $X_{n}^{i, j}$ is less than $2 n^{-1}$. The arc length of $X_{n}$ is greater than $(2 n)$ !.

Suppose that $h: X_{n} \rightarrow X_{m}$ is a homeomorphism for some $n<m<$ $\infty$. Then for some $i=1, \ldots,(2 n) !, j=1, \ldots, n$ and $k=1, \ldots,(2 m)$ !, it must happen that $h\left(X_{n}^{i, j}\right) \supset X_{m}^{k}$. If not, then each $h\left(X_{n}^{i, j}\right)$ meets at most two of the segments $X_{m}^{i}$, so the arc length of each mapped segment $h\left(X_{n}^{i, j}\right)$ is less than 4 . The arc length of $h\left(X_{n}\right)$, which is the sum of the arc lengths of the $(2 n) ! n$ mapped segments $h\left(X_{n}^{i, j}\right)$, is less than $4(2 n) ! n$. Since $4(2 n) ! n<(2 m)$ !, the map $h$ could not be surjective. It follows that there exists a pair of points $u, v \in X_{n}$ with $d(u, v) \leq 2 n^{-1}$ but with $d(h(u), h(v)) \geq 1$.

Now if $f \in H(X)$, then it follows that the induced homeomorphism $[f]$ on the space $[X]$ is the identity on all but finitely many points. For if not, then by replacing $f$ by $f^{-1}$ if necessary, we can assume that there are sequence $\left(m_{i}\right)$ and $\left(n_{i}\right)$ in $\mathbb{N}$ tending to infinity such that $f\left(X_{n_{i}}\right)=X_{m_{i}}$ and $m_{i}>n_{i}$ for all $i$. Hence, there exist $u_{i}, v_{i} \in X_{n_{i}}$ with $d\left(u_{i}, v_{i}\right) \leq 2 n_{i}^{-1}$ but with $d\left(f\left(u_{i}\right), f\left(v_{i}\right)\right) \geq 1$. Thus, there are convergent subsequences of $\left\{u_{i}\right\}$ and $\left\{v_{i}\right\}$ with a common limit in $X_{\infty}$. Hence, $f$ could not extend to a continuous function on all of $X$.

Thus, there exists $N \in \mathbb{N}$ such that $f\left(X_{n}\right)=X_{n}$ for all $N \leq n \leq \infty$. Since the isolated components $X_{n}$ are invariant for $n$ large enough, $X$ is $f$-decomposable.

Example 3.19. We construct spaces $X$ and $X^{+}$so that

- The isolated components of $X$ and $X^{+}$are all homeomorphic to one another and their union is dense. 
- $X^{+}$is $H\left(X^{+}\right)$-indecomposable but it is $f$-decomposable for all $f \in H\left(X^{+}\right)$.

- There exists $f \in H(X)$ such that $X$ is $f$-indecomposable, but no $f \in H(X)$ is chain transitive.

Let $Z=\left\{x_{n}: n \in \mathbb{Z}\right\} \subset I=[0,1]$ with

$$
x_{n}= \begin{cases}1-(n+2)^{-1} & \text { for } n=0,1, \ldots, \\ (|n|+2)^{-1} & \text { for } n=-1,-2, \ldots\end{cases}
$$

Define for $n \in \mathbb{Z}$

$$
\begin{gathered}
a_{n}=\left(x_{n}, 0\right), \quad b_{n}=\left(x_{n},(|n|+1)^{-1}\right), \\
I_{n}=\left\{\left(x_{n}, t\right): 0 \leq t \leq(|n|+1)^{-1}\right\} .
\end{gathered}
$$

Define

$$
\begin{gathered}
J=I \times\{0\}, \quad H=[-1,0] \times\{0\} \\
C=H \cup J \cup \bigcup\left\{I_{n}: n \in \mathbb{Z}\right\}, \\
Z_{0}=\left\{a_{n}: n \in \mathbb{Z}\right\}, \quad Z_{1}=\left\{b_{n}: n \in \mathbb{Z}\right\}, \\
e_{0}=(0,0), \quad e_{1}=(1,0), \quad e_{-1}=(-1,0) .
\end{gathered}
$$

We will call $C$ a $c o m b$ with handle $H$.

The group $H(C)$ fixes $e_{-1}, e_{0}$ and $e_{1}$. Each of the sets $Z_{0}$ and $Z_{1}$ is a single $H(C)$-orbit. The closed sets $J$ and $H$ are $H(X)$-invariant.

If $h \in H(C)$, then

$$
h\left(b_{n}\right)=b_{n+k} \Leftrightarrow h\left(a_{n}\right)=a_{n+k} \Leftrightarrow h\left(I_{n}\right)=I_{n+k} .
$$

In that case $h\left(a_{n \pm 1}\right)=a_{n \pm 1+k}$, and so $h$ induces a translation by $k$ on the sequences $Z_{0}$ and $Z_{1}$. If $k>0$, then $e_{1}$ is an attractor with complementary repellor $H$, and the reverse is true if $k<0$. If $k=0$, then $h$ fixes each point of $Z_{0}$ and of $Z_{1}$.

On $C$ define $T$ by

$$
\begin{aligned}
& T\left(e_{0}\right)=e_{0}, \quad T\left(e_{ \pm 1}\right)=e_{ \pm 1}, \\
& T\left(a_{n}\right)=a_{n+1}, \quad T\left(b_{n}\right)=b_{n+1},
\end{aligned}
$$

and with $T:\left[a_{n-1}, a_{n}\right] \rightarrow\left[a_{n}, a_{n+1}\right], T: I_{n} \rightarrow I_{n+1}$ and $T: H \rightarrow H$ linear for all $n \in \mathbb{Z}$. Thus, $T \in H(C)$ induces a translation by 1 on $Z_{0}$ and $Z_{1}$.

For $n \in \mathbb{Z}$ let

$$
\begin{aligned}
I_{n}^{*} & =I_{n} \cup\left\{\left(x,(|n|+1)^{-1}\right): \frac{x_{n-1}+x_{n}}{2} \leq x \leq \frac{x_{n+1}+x_{n}}{2}\right\}, \\
C_{n} & =C \cup I_{n}^{*},
\end{aligned}
$$

a comb with a queer tooth $I_{n}^{*}$ at $n$ replacing $I_{n}$. 
Observe that $C$ and each $C_{n}$ are connected.

If $f: C_{n} \rightarrow C_{m}$ is a homeomorphism, then $f\left(I_{n}^{*}\right)=I_{m}^{*}$. If $h \in H(C)$, then $h$ extends to a homeomorphism from $C_{n}$ to $C_{m}$ iff $h\left(I_{n}\right)=I_{m}$. Thus, $C_{n}$ and $C_{m}$ are homeomorphic for all $n, m \in \mathbb{Z}$.

In $\mathbb{R}^{2} \times I$ we define

$$
\begin{aligned}
X & =C \times\{0,1\} \cup \bigcup\left\{C_{n} \times\left\{x_{n}\right\}: n \in \mathbb{Z}\right\}, \\
X^{+} & =C \times\{1\} \cup \bigcup\left\{C_{n} \times\left\{x_{n}\right\}: n \in \mathbb{N}\right\},
\end{aligned}
$$

Thus, $X$ and $X^{+}$both have a dense union of isolated components, and each isolated component is homeomorphic to $C_{0}$. It follows from Lemma 3.15 that $X$ is $H(X)$-indecomposable and $X^{+}$is $H\left(X^{+}\right)$-indecomposable.

Any homeomorphism in $H\left(X^{+}\right)$restricts to a homeomorphism of $C \times\{1\}$ and so restricts to translation by $k$ on $Z_{0} \times\{1\}$. This means that $h$ must map $C_{n} \times\left\{x_{n}\right\}$ to $C_{n+k} \times\left\{x_{n+k}\right\}$ when $n \geq N$ for $N$ sufficiently large, and we may suppose $N+k>0$. This implies that $h$ maps the set of $N$ complementary components $\left\{C_{n} \times\left\{x_{n}\right\}: 0 \leq n<N\right\}$ to the set of $N+k$ components $\left\{C_{n} \times\left\{x_{n}\right\}: 0 \leq n<N+k\right\}$. This requires that $k=0$. Hence, each isolated component $C_{n} \times\left\{x_{n}\right\}$ with $n \geq N$ is invariant, and so $X^{+}$is $h$-decomposable.

Thus, $X^{+}$is an example of a space which is $H\left(X^{+}\right)$-indecomposable but such that $X^{+}$is $h$-decomposable for every $h \in H\left(X^{+}\right)$.

In the case of $X$, we start by assuming that $h$ fixes each of the two non-isolated components, i.e. $h$ maps $C \times\{\epsilon\}$ to itself for $\epsilon=0,1$. As before, since $h$ translates $Z_{0} \times\{\epsilon\}$ by some $k_{\epsilon}$ for $\epsilon=0,1$, there exists $N \in \mathbb{N}$ large enough that $h$ maps $C_{n} \times\left\{x_{n}\right\}$ to $C_{n+k_{1}} \times\left\{x_{n+k_{1}}\right\}$ for $n \geq N$ and $C_{n} \times\left\{x_{n}\right\}$ to $C_{n+k_{0}} \times\left\{x_{n+k_{0}}\right\}$ for $-n \geq N$. Again we can choose $N$ large enough that $N+k_{\epsilon}>0$ for $\epsilon=0,1$. This implies that $h$ maps the set of complementary components $C_{n} \times\left\{x_{n}\right\}$ for $-N<n<N$ to the set of components $C_{n} \times\left\{x_{n}\right\}$ for $-N+k_{0}<n<N+k_{1}$. This requires that $k_{0}=k_{1}$, and we let $k=k_{0}=k_{1}$.

If $k>0$, then $C \times\{1\}$ is an attractor and $C \times\{0\}$ is a repellor with the reverse if $k<0$. Hence, $h$ is not chain transitive. If $k=0$, then each isolated component $C_{n} \times\left\{x_{n}\right\}$ is invariant for $|n| \geq N$, and so $X$ is $h$-decomposable.

The remaining possibility is that $h$ interchanges the two limit components $C \times\{\epsilon\}$ for $\epsilon=0,1$ and then each is invariant for $h^{2}$. Applying the previous argument to $h^{2}$, we see that for a large $N$ the components are translated by $h^{2}$ with a common $k$. If $k>0$, then $C \times\{1\}$ is an attractor for $h^{2}$ while $C \times\{0\}$ is a repellor. But since $h$ commutes with $h^{2}$ this would imply that $C \times\{0\}=h(C \times\{1\})$ would be an 
attractor for $h^{2}$ as well, which it is not. Similarly, $k<0$ leads to a contradiction. It follows that in this interchange case $k=0$, and so $C_{n} \times\left\{x_{n}\right\}$ is $h^{2}$-invariant for $|n|$ sufficiently large. Hence, for each such $n$ the set $C_{n} \times\left\{x_{n}\right\} \cup h\left(C_{n} \times\left\{x_{n}\right\}\right)$ is clopen and $h$-invariant, so $X$ is $h$-decomposable when $h$ interchanges the ends.

Finally, extend $T: C \rightarrow C$ by $T_{n}: C_{n} \times\left\{x_{n}\right\} \rightarrow C_{n+1} \times\left\{x_{n+1}\right\}$ for all $n \in \mathbb{Z}$ and by $T \times 1_{\{0,1\}}$ on $C \times\{0,1\}$ to obtain a homeomorphism (with $k=1$ ) with respect to which $X$ is not decomposable.

Thus, $X$ is an example of a space with an element $h \in H(X)$ so that $X$ is not $h$-decomposable, but nonetheless there is no chain transitive homeomorphism in $H(X)$.

\section{Spaces with All Homeomorphisms Chain Transitive}

Having considered spaces which admit no chain transitive homeomorphisms, we turn to the opposite extreme to consider spaces such that every homeomorphism is chain transitive. By Proposition 2.7 (e) the identity $1_{X}$ is chain transitive iff $X$ is connected, and if $f \in H(X)$ with $X$ connected, then $f$ is chain transitive iff it is chain recurrent.

A space $X$ is called rigid if $1_{X}$ is the only homeomorphism on $X$, i.e. the group $H(X)$ is trivial. Such spaces were introduced and constructed by de Groot and Willie [11. For a connected rigid space the only element of $H(X)$ is chain transitive. We construct some more interesting examples by using rigid spaces as tools. We need a pairwise disjoint sequence $\left\{Z_{n}\right\}$ of connected, locally connected spaces such that

(*) For any nonempty open subset $U$ of $Z_{n}$ and any disk $I^{k}$, there does not exists a homeomorphism of $U \times I^{k}$ onto any subset of $\left(Z_{n} \backslash U\right) \times I^{k}$ or onto any subset of $Z_{m} \times I^{k}$ with $m \neq n$.

Furthermore, for every $n \in \mathbb{N}$ and for any finite $F \subset Z_{n}$, the set $Z_{n} \backslash F$ has only finitely many components and for any positive integer $N$ there is a subset $F \subset Z_{n}$ of cardinality $N$ such that $Z_{n} \backslash F$ is connected.

Condition $(*)$ is a slight strengthening of the condition on a space called strongly chaotic in [8]. We construct such a sequence in the Appendix, Section 5. For each $Z_{n}$ we choose a pair of distinct points $e_{n}^{-}, e_{n}^{+} \in Z_{n}$. Our examples are obtained by using such rigid spaces instead of the unit interval in some common constructions.

Example 4.1. We can think of the real line as a graph with $\mathbb{Z}$ as the set of vertices and intervals $[n, n+1]$ as edges. Now let $Z$ be one of the chaotic spaces described above with points $e^{-}, e^{+} \in Z$. Let $X_{0}$ be the quotient space of $\mathbb{Z} \times Z$ with $\left(n, e^{+}\right)$identified with $\left(n+1, e^{-}\right)$. If $t$ is the translation homeomorphism on $\mathbb{Z}$ with $t(n)=n+1$, then $t$ has 
a unique extension $t$ to $X_{0}$ which is the quotient of $t \times 1_{Z}$. The only homeomorphisms on $X_{0}$ are the iterates $t^{n}$. Let $X$ be the one-point compactification of $X_{0}$ with the additional point $\infty$. Let $t \in H(X)$ be the unique homeomorphism extension of $t$ on $X_{0}$ and so of $t$ on $\mathbb{Z}$. Since $X$ is connected, $1_{X}$ is chain transitive. For any $n \neq 0$, we have $\{\infty\}=\omega\left(t^{n}\right)(x)=\alpha\left(t^{n}\right)(x)$ for all $x \in X$, and so $t^{n}$ is chain transitive. In this case, $H(X)$ is isomorphic to $\mathbb{Z}$.

In general, suppose that $G$ is a finitely generated (and hence countable) group. Following de Groot [10] we let $X_{0}$ be the Cayley graph with rigid spaces as linking edges. That is, let $\left\{g_{1}, . ., g_{n}\right\}$ be a list of generators for $G$, and let $\left\{Z_{1}, \ldots, Z_{n}\right\}$ be distinct strongly chaotic spaces as above, with chosen pairs of points. Let $X_{0}$ be the quotient space of $G \cup\left[G \times\left(\bigcup_{i=1}^{n} Z_{i}\right)\right]$ with $\left(g, e_{i}^{+}\right)$identified with $\left(g_{i} g, e_{i}^{-}\right)$for $g \in G, i=1, \ldots, n$ and $\left(g, e_{i}^{-}\right)$identified with $g \in G$ for $i=1, \ldots, n$. Because there are only finitely many generators, the space $X_{0}$ is locally compact and the set of vertices $\{g \in G\}$ is invariant with respect to any homeomorphism $h$. Furthermore, if $g \in G$, then $h\left(g_{i} g\right)=g_{i} h(g)$, and so $h$ commutes with all left translations. It follows that, on $G$, the mapping $h$ is the right translation $r_{v}$, where $v=h(u)$ and $u$ is the identity element of $G$. Thus, on $X_{0}$, the mapping $h$ is the quotient of the map $r_{v} \cup\left[r_{v} \times 1_{\cup_{i} Z_{i}}\right]$. Let $X$ be the one-point compactification of $X_{0}$, and let $r_{v}$ denote the extension of $h$ to $X$. If $v$ is of finite order $k$, then $\left(r_{v}\right)^{k}=1_{X}$ and so $r_{v}$ is chain transitive on $X$. If $v$ is of infinite order, then $\{\infty\}=\omega\left(r_{v}\right)(x)=\alpha\left(r_{v}\right)(x)$, and so $r_{v}$ is chain transitive on $X$ in this case as well. The group $H(X)$ is isomorphic to the discrete group $G$ by $v \mapsto r_{v}^{-1}$.

In these cases, the homeomorphism group is discrete. It is possible to obtain rather large non-discrete groups. We first review some standard topology constructions.

A pointed space is a pair $(X, x)$ consisting of a space with a chosen base point $x \in X$. We let $H(X, x)$ denote the closed subgroup of $H(X)$ consisting of those homeomorphisms which fix $x$. A space $Y$ can be regarded as a pointed space with base point an isolated point not in $Y$. If $\left(X_{1}, x_{1}\right)$ and $\left(X_{2}, x_{2}\right)$ are pointed spaces and $f: X_{1} \rightarrow X_{2}$ is a function, we use the notation $f:\left(X_{1}, x_{1}\right) \rightarrow\left(X_{2}, x_{2}\right)$ to mean that $f\left(x_{1}\right)=x_{2}$.

If $A$ is a nonempty closed subset of a space $X$, then the space $X / A$ with $A$ smashed to a point is the quotient space of $X$ with respect to the closed equivalence relation $1_{X} \cup A \times A$. Thus, the quotient map $q: X \rightarrow X / A$ is a homeomorphism between the open sets $X \backslash A$ and $X / A \backslash\left\{x_{A}\right\}$ with $x_{A}$ the point which is the image of $A$. 
Given two pointed spaces $\left(X_{1}, x_{1}\right),\left(X_{2}, x_{2}\right)$, their smash product is

$$
\left(X_{1}, x_{1}\right) \#\left(X_{2}, x_{2}\right)=\left(X_{12}, x_{12}\right) \text {, }
$$

a pointed space consisting of the product $X_{1} \times X_{2}$ with the wedge $X_{1} \times\left\{x_{2}\right\} \cup\left\{x_{1}\right\} \times X_{2}$ smashed to the point $x_{12}$. We can also define the smash product of a pointed space $\left(X_{1}, x_{1}\right)$ and any space $X_{2}$ as $\left(X_{1}, x_{1}\right) \# X_{2}=\left(X_{12}, x_{12}\right)$, where $X_{12}$ is the product $X_{1} \times X_{2}$ with $\left\{x_{1}\right\} \times X_{2}$ smashed to the point $x_{12}$. Notice that in this case we can regard the space $X_{12}$ as the one-point compactification of $\left(X_{1} \backslash x_{1}\right) \times X_{2}$. The projections $\pi_{1}:\left(X_{1}, x_{1}\right) \# X_{2} \rightarrow\left(X_{1}, x_{1}\right)$ and $\pi_{2}: X_{1} \backslash\left\{x_{1}\right\} \times X_{2} \rightarrow$ $X_{2}$ are open and surjective.

We can define the smash product of two continuous functions once we fix base points from the domains. For $i=1,2$, let $X_{i}$ and $Y_{i}$ be spaces, $f_{i}: X_{i} \rightarrow Y_{i}$ a continuous function and $x_{i} \in X_{i}$ a base point. We set $y_{i}=f_{i}\left(x_{i}\right)$ to obtain a pointed space $\left(Y_{i}, y_{i}\right)$ for $i=1,2$. Let $\left(X_{12}, x_{12}\right)=\left(X_{1}, x_{1}\right) \#\left(X_{2}, x_{2}\right)$ and $\left(Y_{12}, y_{12}\right)=\left(Y_{1}, y_{1}\right) \#\left(Y_{2}, y_{2}\right)$, and let $q: X_{1} \times X_{2} \rightarrow X_{12}$ and $r: Y_{1} \times Y_{2} \rightarrow Y_{12}$ be the quotient maps. We define a continuous function $f=\left(f_{1}, x_{1}\right) \#\left(f_{2}, x_{2}\right)$ from $\left(X_{12}, x_{12}\right)$ to $\left(Y_{12}, y_{12}\right)$ by the formula $f \circ q=r \circ\left(f_{1} \times f_{2}\right)$. We can also define the smash product of two functions when only one of the domains has a base point. If $(V, v)=\left(X_{1}, x_{1}\right) \# X_{2}$ and $(W, w)=\left(Y_{1}, y_{1}\right) \# Y_{2}$ and if $s: X_{1} \times X_{2} \rightarrow V$ and $t: Y_{1} \times Y_{2} \rightarrow W$ are the quotient maps, then we define the continuous function $g=\left(f_{1}, x_{1}\right) \# f_{2}$ from $(V, v)$ to $(W, w)$ by $g \circ s=t \circ\left(f_{1} \times f_{2}\right)$.

Example 4.2. We construct a space such that every homeomorphism is chain transitive and the homeomorphism group contains a nontrivial path-connected subgroup.

Let $(Z, e)$ be a chaotic space $Z$, as above, with base point $e \in Z$ such that $Z \backslash\{e\}$ is connected. Let $W$ be a connected, compact manifold (perhaps with boundary) of positive dimension. Let $\left(X, e_{X}\right)=$ $(Z, e) \# W$. If $(z, w) \in Z \times W$ and $h$ is a homeomorphism from an open set containing $(z, w)$ into $Z \times W$, then $h(z, w)=\left(z_{1}, w_{1}\right)$ with $z, z_{1} \in Z \backslash\{e\}$ implies $z=z_{1}$. If not, then we can choose disk neighborhoods of $w$ and $w_{1}$ each homeomorphic to $I^{k}$ with $k$ the dimension of $W$, and we can choose disjoint open neighborhoods $U$ of $z$ and $U_{1}$ of $z_{1}$ so that $h$ induces a homeomorphism from $U \times I^{k}$ onto a subset of $U_{1} \times I^{k}$, and this contradicts Condition $(*)$. If $h(z, w)=e_{X}$ with $z \in Z \backslash\{e\}$, then $h$ would map points $\left(z_{2}, w_{2}\right)$ close to $(z, w)$ to points $\left(z_{1}, w_{1}\right)$ with $z_{1}$ close to $e$. This does not happen by the previous argument. Thus, it follows that $\pi_{1} \circ h=\pi_{1}$, where $\pi_{1}$ is the projection to $(Z, e)$. This implies that $H(X)=H\left(X, e_{X}\right)$, and $\pi_{1}$ maps every $h \in H(X)$ to $1_{Z}$. 
Since the homeomorphisms of $X$ leave the preimages of $\pi_{1}$ invariant, it follows that every $h \in H(X)$ is of the form $h(z, w)=(z, q(z)(w))$ with $q: Z \backslash\{e\} \rightarrow H(W)$ a continuous map. Thus, the space of continuous maps $C(Z \backslash\{e\}, H(W))$ with the obvious group structure is isomorphic as a group with $H(X)$. Notice that we need not worry about behavior as $z$ approaches $e$ in $Z$ since all of $\{e\} \times W$ is smashed to a point. Thus, the isomorphism is topological if we choose an increasing sequence $\left\{K_{n}\right\}$ of compacta in $Z$ with union $Z \backslash\{e\}$ and define the metric $d\left(q_{1}, q_{2}\right)=\sup _{n} 2^{-n} d_{H}\left(\left.q_{1}\right|_{K_{n}},\left.q_{2}\right|_{K_{n}}\right)$ with $d_{H}$ the uniform metric on $H(W)$.

In particular, the constant maps $q$ yield $H(W)$ as a subgroup of $H(X)$. Since $W$ is a manifold of positive dimension, it follows that the path component of the identity in $H(W)$ and hence in $H(X)$ are nontrivial subgroups. The remaining path components are cosets and so are nontrivial as well.

Let $h \in H(X)$. Given $\epsilon>0$ and $(z, w) \in X \backslash\left\{e_{X}\right\}$, there is an $\epsilon$-chain $z_{0}, \ldots, z_{N}$ for $1_{Z}$ with $z_{0}=z$ and $z_{N}=e$ and $z_{i} \neq e$ for $i<N$. That is, $d\left(z_{n+1}, z_{n}\right) \leq \epsilon$. Define $\left\{w_{0}, \ldots, w_{N}\right\}$ by $w_{0}=w$ and $h\left(z_{i}, w_{i}\right)=\left(z_{i}, w_{i+1}\right)$ for $i<N$. Clearly, $\left(z_{0}, w_{0}\right), \ldots,\left(z_{N-1}, w_{N-1}\right), e_{X}$ is an $\epsilon$-chain for $h$ from $(z, w)$ to $e_{X}$. Similarly, there is an $\epsilon$-chain for $h^{-1}$ from $(z, w)$ to $e_{X}$. It follows that $h$ is chain recurrent. Since $X$ is connected, $h$ is chain transitive.

Now let $(Y, e)$ be a pointed space such that $Y$ and $Y \backslash\{e\}$ are connected and, for every $y \in Y$, the open set $Y \backslash\{y\}$ has only finitely many components. Let $C$ be a zero-dimensional space, and let $\left(X, e_{X}\right)=$ $(Y, e) \# C$. By assumption on $Y$, the set $X \backslash\{(y, c)\}$ has only finitely many components for $y \in Y \backslash\{e\}, c \in C$. Since $C$ is zero-dimensional, the components of $X \backslash\left\{e_{X}\right\}$ are the sets $\{(Y \backslash\{e\}) \times\{c\}: c \in C\}$. Thus, if $C$ is infinite, $X \backslash\left\{e_{X}\right\}$ has infinitely many components, while $X \backslash\{(y, c)\}$ has only finitely many components for $y \in Y \backslash\{e\}, c \in C$. Hence, if $C$ is infinite, then any homeomorphism of $X$ fixes $e_{X}$. We will assume that, even with $C$ finite, the space $Y$ is such that the point $e_{X}$ is fixed by every homeomorphism of $X$. It follows that for any homeomorphism on $h$ on $X$, the projection $\pi_{2}: X \backslash\left\{e_{X}\right\} \rightarrow C$ maps the restriction of $h$ on $X \backslash\left\{e_{X}\right\}$ to a homeomorphism on $C$. Thus, we obtain $\left(\pi_{2}\right)_{*}: H(X) \rightarrow H(C)$ a continuous, surjective homomorphism of topological groups. This splits via the continuous injection $j: H(C) \rightarrow H(X)$ given by $j(k)=\left(1_{Y}, e\right) \# k$. Now suppose that $h \in H(X)$ is in the kernel of $\left(\pi_{2}\right)_{*}$, that is, it projects to $1_{C}$. This means that every $(Y \backslash\{e\}) \times\{c\}$ is $h$-invariant. It follows that $h(y, c)=(q(c)(y), c)$, where $q: C \rightarrow H(Y, e)$ is a continuous 
map. That is, the kernel is $C(C, H(Y, e))$ with the obvious topological group structure. Thus, $H(X)$ is the semi-direct product of $H(C)$ with $C(C, H(Y, e))$. The adjoint action of $j(H(C))$ is just the action $H(C) \times C(C, H(Y, e)) \rightarrow C(C, H(Y, e))$ given by $(k, q) \mapsto q \circ\left(k^{-1}\right)$.

Example 4.3. We construct a space such that every homeomorphism is chain transitive and the homeomorphism group is isomorphic to the homeomorphism group of the Cantor set.

Let $(Z, e)$ be a pointed chaotic space as before, and let $\left(X, e_{X}\right)=$ $(Z, e) \# C$ with $C$ a zero-dimensional space. We first check that even if $C$ is finite, any homeomorphism $h$ on $X$ fixes $e_{X}$. If not, then there exist points $x_{1}, x_{2} \in Z$, distinct from each other and distinct from $e$, and points $a_{1}, a_{2} \in C$ such that $h\left(x_{1}, a_{1}\right)=\left(x_{2}, a_{2}\right)$. This implies $h$ induces a homeomorphism between sufficiently small neighborhoods $U_{1}$ of $x_{1}$ and $U_{2}$ of $x_{2}$. Choosing these as disjoint neighborhoods, we obtain a contradiction of Condition $(*)$.

In this case $H(Z, e)=H(Z)=\left\{1_{Z}\right\}$. That is, the group $H(Z, e)$ is trivial, and so the group $C(C, H(Z, e))$ is trivial. This means that $\left(p_{2}\right)_{*}: H(X) \rightarrow H(C)$ and $j: H(C) \rightarrow H(X)$ are inverse isomorphisms, so every homeomorphism on $X$ is mapped by $\pi_{1}$ to $1_{Z}$. Just as in Example 4.2, it follows that every homeomorphism is chain transitive.

When $C$ is a Cantor set, we obtain an example with homeomorphism group isomorphic to the homeomorphism group of the Cantor set.

Because of the rigidity of the connecting links, it is not true in these examples that $H(X)$ acts transitively on $X$. We can obtain examples which satisfy this additional condition by using the beautiful construction of Slovak spaces due to Downarowicz, Snoha, and Tywoniuk in [13.

Let $g$ be a totally transitive homeomorphism on a Cantor set $W$. That is, $g^{n}$ is topologically transitive for all $n \in \mathbb{Z} \backslash\{0\}$. The construction begins with the suspension of $g$. That is, let $Y=W \times[0,1]$ with $(x, 1)$ identified with $(g(x), 0)$ for all $x \in W$. On $Y$ we define the real flow $\phi: \mathbb{R} \times Y \rightarrow Y$, the associated time $t$ map $\phi^{t}: Y \rightarrow Y$, and the path map $\phi_{x}: \mathbb{R} \rightarrow Y$ for $t \in \mathbb{R}, x \in W$ by

$$
\begin{aligned}
\phi(t,(x, s))= & \phi^{t}(x, s)=\left(g^{[t+s]}(x),\{t+s\}\right), \\
& \phi_{x}(t)=\phi^{t}(x, 0),
\end{aligned}
$$

where $[a]$ and $\{a\}$ are the integer part and fractional part, respectively, of the real number $a$. Identifying $W$ with $W \times\{0\} \subset Y$, we see that $g$ on $W$ is identified with the time-one map $\phi^{1}$ restricted to $W$. 
Observe that the flow $\phi$ restricts to a homeomorphism from $\left[-\frac{1}{3}, \frac{1}{3}\right] \times$ $W$ onto a neighborhood of $W \times\{s\}$ in $Y$ for $s \in[0,1]$. It follows that the path components of $Y$ are exactly the $\mathbb{R}$-orbits of the flow. For $x \in W$ we let $\mathbb{R} x=\phi_{x}(\mathbb{R})$ denote the $\mathbb{R}$-orbit through $(x, 0)$.

In $Y$ there are three types of path components:

Type 1 If $x$ is a periodic point for $g$, then $\mathbb{R} x$ is an circle embedded in $Y_{0}$. This is a circle type path component.

Type 2 If $x$ is neither recurrent for $g$ nor for $g^{-1}$, i.e. $x \notin \omega g(x) \cup \alpha g(x)$, then $\mathbb{R} x$ is an embedded copy of $\mathbb{R}$. That is, $\phi_{x}$ is a homeomorphism from $\mathbb{R}$ onto its image in $Y$. This is an embedded $\mathbb{R}$ type path component.

Type 3 If $x$ is not periodic but $x \in \omega g(x) \cup \alpha g(x)$, then $\phi_{x}$ is a continuous injection which is not a homeomorphism onto its image. In fact, $\mathbb{R} x \subset Y_{0}$ is not locally connected. This is an injected $\mathbb{R}$ type path component.

The Slovak space construction is based on the following result:

Theorem 4.4 ([13, Lemma 4.3]). Let $f$ be a homeomorphism on a space $Y$ with $y_{0}$ a point of $Y$ which is not a periodic point for $f$. Let $\left\{a_{n}: n \in \mathbb{Z}\right\}$ be a sequence of positive reals such that $\Sigma_{n} a_{n}=1$ and the set $\left\{\left|\ln \left(a_{n}\right)-\ln \left(a_{n-1}\right)\right|: n \in \mathbb{Z}\right\}$ is bounded, and let $u: Y \backslash\left\{y_{0}\right\} \rightarrow[0,1]$ be a continuous function. Let $Y^{\prime}=Y \backslash \mathcal{O} f_{ \pm}\left(y_{0}\right)$, and on $Y^{\prime}$ define the continuous function $u^{\prime}=\Sigma_{n} a_{n} u \circ f^{n}$ so that $u^{\prime}$ is a closed subset of $Y^{\prime} \times$ $[0,1]$ with the first coordinate projection $\pi: u^{\prime} \rightarrow Y^{\prime}$ a homeomorphism. Define on the set $u^{\prime}$ the homeomorphism $f^{\prime}=(\pi)^{-1} \circ f \circ(\pi)$. Let $X$ be the closure of $u^{\prime}$ in $Y \times[0,1]$.

The homeomorphism $f^{\prime}$ and its inverse are uniformly continuous on $u^{\prime}$, and so $f^{\prime}$ extends to a homeomorphism $h$ on $X$. The first coordinate projection $\pi: X \rightarrow Y$ maps $h$ on $X$ to $f$ on $Y$. If $y \in Y^{\prime}$, then $\left(y, u^{\prime}(y)\right)$ is the unique point of $X$ which is mapped by $\pi$ to $y$. Hence, $h$ is an almost one-to-one extension of $f$.

Now, following [13], we apply this construction to the situation above.

Let $x_{0}$ be an element of the dense $G_{\delta}$ set $\bigcap\left\{\operatorname{Trans}\left(g^{n}\right): n \in \mathbb{Z} \backslash\{0\}\right\}$. By [4] Proposition 6.3 (a) the set of $\tau \in(0, \infty)$ such that $\omega \phi^{n \tau}\left(x_{0}\right)=$ $Y=\alpha \phi^{n \tau}\left(x_{0}\right)$ is residual in $(0, \infty)$. Fix such a $\tau \in(0,1)$, and let $f=\phi^{\tau}$. Thus, $x_{0}$ is a transitive point for $f^{n}$ on $Y$ for all $n \in \mathbb{Z} \backslash\{0\}$.

We first define $u$ on a piece of the orbit of the point $y_{0}=\left(x_{0}, 0\right)$ :

$$
u\left(\phi_{x_{0}}(t)\right)= \begin{cases}0 & \text { for }-\frac{1}{2} \leq t<0 \\ \frac{1}{2}\left(1-\cos \left(\frac{\pi}{t}\right)\right) & \text { for } 0<t \leq \frac{1}{2}\end{cases}
$$


Apply the Tietze Extension Theorem to obtain the continuous function $u: Y \backslash\left\{y_{0}\right\} \rightarrow[0,1]$.

Apply Theorem 4.4 to $Y$ with the homeomorphism $f$. We obtain an almost one-to-one lift $h$ on $X=\overline{u^{\prime}} \subset Y \times[0,1]$. All of the path components of $X$ are mapped by $\pi$ homeomorphically onto the path components of $Y$, except that the Type 3 path component $\mathbb{R} x_{0}$ is cut into a sequence of path components of a new type.

Type 4 The path component Comp $p_{n}$ of $h^{n}\left(y_{0}\right)$ is mapped by $\pi$ onto the set $\phi_{x_{0}}(((n-1) \tau, n \tau])$. As $t \searrow(n-1) \tau$, above the open interval end, there is a topologist's sine which projects homeomorphically. Above the $n \tau$ endpoint there is a vertical segment $J_{n}=$ $\left\{h^{n}\left(y_{0}\right)\right\} \times\left[0, a_{-n}\right]$ to which the oscillating end of the path component $\operatorname{Comp}_{n+1}$ converges. That is, $J_{n}=\operatorname{Comp}_{n} \cap \overline{C_{o m p}}$. Thus, each path component $C_{o m p}$ is a homeomorphic image of $\mathbb{R}_{+}=[0, \infty)$. Each is an embedded $\mathbb{R}_{+}$path component.

Theorem 4.5. The homeomorphism group of $X$ is $H(X)=\left\{h^{n}: n \in\right.$ $\mathbb{Z}\}$. For all $n \neq 0$ the homeomorphism $h^{n}$ is topologically transitive.

Proof: If $n \neq 0$, then by choice of $\tau$, the homeomorphism $f^{n}=\phi^{n \tau}$ is topologically transitive on $Y$. Because $\pi$ mapping $h$ to $f$ is an almost one-to-one lift, it follows that $h^{n}$ is topologically transitive on $X$.

If $h_{1}$ is any homeomorphism on $X$, then the Type 4 component $C o m p_{n}$ is mapped to some Type 4 component Comp $p_{n+k}$. Furthermore, $J_{n}=\operatorname{Comp}_{n} \cap \overline{C o m p}_{n+1}$ is mapped to $J_{n+k}$. It follows that $\pi$ projects $h_{1}$ to a continuous map on $Y$ which agrees with $f^{k}$ on the dense set $\mathcal{O}\left(f_{ \pm}\right)\left(y_{0}\right)$. It follows that it projects to $f^{k}$. Since $\pi$ is almost one-toone, it follows that $h_{1}=h^{k}$.

Downarowicz, Snoha, and Tywoniuk begin with $g$ on $W$ minimal and observe that, for a residual set of positive reals $\tau$, the homeomorphism $\phi^{\tau}$ is minimal on $Y$. Choosing one such $\tau$, they have $f$ minimal on $Y$. All of the components of $Y$ are then of Type 3 . Then $h^{n}$ is minimal on $X$ for all $n \neq 0$.

We recall and extend their definition of a Slovak space.

Definition 4.6. (a) A space $X$ is a Slovak space if $X$ contains at least three points, $H(X)$ is isomorphic to $\mathbb{Z}$ and every $h \in H(X) \backslash\left\{1_{X}\right\}$ is minimal.

(b) A space $X$ is Slovakian if $X$ contains at least three points, $H(X)$ is nontrivial and every $h \in H(X) \backslash\left\{1_{X}\right\}$ is topologically transitive.

We extend Theorem 4 of [13] with essentially the same proof. 
Theorem 4.7. A Slovakian space is connected, and its homeomorphism group has no elements of finite order other than the identity.

Proof: Let $h$ be a topologically transitive homeomorphism on a space $X$. Suppose that $X$ contains a proper, clopen, nonempty subset $A$. If $h^{-1}(A) \subset A$, then for any $x \in \operatorname{Trans}(h)$, we have $h^{n}(x) \in A$ for some $n \in \mathbb{N}$, and so $x \in A$. Thus, the dense set $\operatorname{Trans}(h)$ is contained in $A$, contradicting the assumption that $A$ is a proper clopen set. It follows that $B=h^{-1}(A) \backslash A$ is a proper, clopen, nonempty set, and $B \cap h(B)=\emptyset$. Define

$$
g(x)= \begin{cases}h(x) & \text { for } x \in B, \\ h^{-1}(x) & \text { for } x \in h(B), \\ x & \text { for } x \in X \backslash(B \cup h(B)) .\end{cases}
$$

The points of the nonempty set $B \cup h(B)$ are periodic with period 2, and so $g \neq 1_{X}$. Since $g^{2}=1_{X}$, it is clear that $g$ is not topologically transitive.

Since $X$ is nontrivial and connected, it is perfect and therefore uncountable. On such a space, no topologically transitive homeomorphism has finite order.

Questions: Does there exists a Slovakian space $X$ for which $H(X)$ is not discrete? More generally, does there exist a nontrivial space $X$ such that the topologically transitive homeomorphisms are dense in $H(X)$ ? If $X$ is such a space, then every $h \in H(X)$ is chain transitive. In particular, since $1_{X}$ is chain transitive, $X$ is connected. On the other hand, $1_{X}$ is not topologically transitive, but it is a limit of topologically transitive homeomorphisms, and so $H(X)$ is not discrete.

The only Slovakian spaces we know of are variations on the original construction of [13]. All of these have homeomorphism group isomorphic to $\mathbb{Z}$.

Now we extend the above construction which was built on a totally transitive homeomorphism $g$ on a Cantor space $W$. Suppose that $B$ is a proper, closed, $g$-invariant subset of $W$ and that $r: B \rightarrow A$ is a continuous surjection which maps $g$ on $B$ to $1_{A}$, the identity on the space $A$. That is, $r^{-1}(a)$ is a closed, invariant set in $B$ for every $a \in A$. Since $B$ is proper, closed and invariant, it is disjoint from $\operatorname{Trans}(g)$. In particular, $x_{0} \notin B$.

Let $\widehat{B}$ be the quotient of $B \times[0,1]$ in $Y$. Since $r \circ g=r$ on $B$, it follows that $r \circ \pi_{1}: B \times[0,1] \rightarrow A$ factors to define the surjection $\widehat{r}: \widehat{B} \rightarrow A$, and each $\widehat{r}^{-1}(a)$ is a $\phi$-invariant closed subset of $Y$. The 
preimage of $\widehat{B}$ via the homeomorphism $\pi: u^{\prime} \rightarrow Y^{\prime}$ is a compact $f^{\prime}$ invariant subset of $Y$. Thus, $\pi^{-1}(\widehat{B})$ is a closed $h$-invariant subset of $X$. Define

$$
\begin{aligned}
& E_{r, Y}=1_{Y} \cup\left[(\widehat{r})^{-1} \circ \widehat{r}\right], \\
& E_{r, X}=1_{X} \cup\left[(\widehat{r} \circ \pi)^{-1} \circ(\widehat{r} \circ \pi)\right],
\end{aligned}
$$

closed equivalence relations on $Y$ and $X$, respectively. Let $q_{r}^{Y}: Y \rightarrow Y_{r}$ and $q_{r}^{X}: X \rightarrow X_{r}$ be the projections to the quotient spaces. The homeomorphisms $f$ and $h$ induce homeomorphisms $f_{r}$ and $h_{r}$ on the quotient spaces. The projection $\pi: X \rightarrow Y$ induces $\pi_{r}: X_{r} \rightarrow Y_{r}$, a continuous, almost one-to-one surjection which maps $h_{r}$ to $f_{r}$. We will regard the homeomorphisms induced by $\widehat{r}: \widehat{B} \rightarrow A$ and $\widehat{r} \circ \pi$ : $\pi^{-1}(\widehat{B}) \rightarrow A$ as identifications, so $A$ is thought of as a subset of $Y_{r}$ and also as a subset of $X_{r}$. These are the sets of fixed points for $f_{r}$ and $h_{r}$, respectively.

For $a \in A$ and $x \in W$, we say that $q_{r}^{Y}(\mathbb{R} x)$ is an a-orbit if $\omega g(x) \subset$ $r^{-1}(a)$ or $\alpha g(x) \subset r^{-1}(a)$. If $\omega g(x) \subset r^{-1}(a)$, then the map $q_{r}^{Y} \circ \phi_{x}$ : $\mathbb{R} \rightarrow Y_{r}$ extends continuously to $\mathbb{R} \cup\{+\infty\}$ by mapping $+\infty$ to $a$. If $x \in B$, then $x$ is an $a$-orbit iff $r(x)=a$, in which case $q_{r}^{Y}(\mathbb{R} x)=\{a\}$. If $K_{1}$ and $K_{2}$ are path components of $A$, they are linked if there exists an $x \in W$ which is both an $a_{1}$-orbit and an $a_{2}$-orbit for some $a_{1} \in$ $K_{1}, a_{2} \in K_{2}$, i.e. if $\alpha g(x) \subset r^{-1}\left(a_{1}\right)$ and $\omega g(x) \subset r^{-1}\left(a_{2}\right)$ or vice-versa. Two path components are linkage equivalent if there is a finite sequence $K_{1}, \ldots, K_{N}$ joining them with each $K_{i}$ linked to its successor.

In $Y_{r}$ we have a new path component type:

Type 5 Let $[K]$ be a linkage equivalence class of path components of $A$. The $[K]$-component in $Y$ is the union of all of the $a$-orbits for $a \in K_{1} \in[K]$ and of the path components $K_{1} \in[K]$.

It is possible for a $[K]$-component to be of embedded $\mathbb{R}_{+}$type. The only way this can happen is if the embedding of $[0, \infty)$ onto the $[K]$ component maps 0 to a point of $A$. The endpoint of this $\mathbb{R}_{+}$type component lies in $A$.

Theorem 4.8. The homeomorphism group of $X_{r}$ is $H\left(X_{r}\right)=\left\{h_{r}^{n}: n \in\right.$ $\mathbb{Z}\}$. The homeomorphism $h_{r}^{n}$ is topologically transitive for all $n \neq 0$.

Proof: The surjection $q_{r}^{X}$ maps the totally transitive homeomorphism $h$ onto the totally transitive homeomorphism $h_{r}$.

If $h_{1}$ is any homeomorphism on $X_{r}$, we proceed just as in the proof of Theorem 4.5. There is, however, one tricky bit. It is possible that a Type 5 path component is of embedded $\mathbb{R}_{+}$type. If $C o m p_{n}$ maps to 
$\operatorname{Comp}_{n+k}$, then just as before, $J_{n}=\operatorname{Comp}_{n} \cap \overline{\operatorname{Comp}_{n+1}}$ is mapped to $J_{n+k}$, and $h_{1}=h^{k}$ as before.

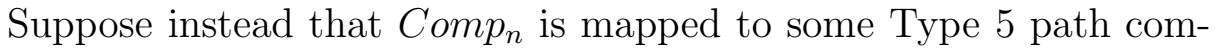
ponent, which we call $Q_{n}$. Then $C o m p_{n+1}$ will have to be mapped to a Type 5 path component $Q_{n+1}$ with $J_{n}$ mapping to $Q_{n} \cap \overline{Q_{n+1}}$, and this set contains the endpoint of $Q_{n}$ which is in $A$. Thus, there is a sequence $\left\{x_{n} \in J_{n}: n \in \mathbb{Z}\right\}$ with $h_{1}\left(x_{n}\right) \in A$. But the sequence $\left\{x_{n}\right\}$ projects to the $f_{r}$-orbit of $y_{0}$, and this is dense in $Y_{r}$. Since $\pi_{r}: X_{r} \rightarrow Y_{r}$ is an almost one-to-one map, the sequence $\left\{x_{n}\right\}$ is dense in $X_{r}$. Since the proper closed subset $A$ contains the sequence $\left\{h_{1}\left(x_{n}\right)\right\}$ and $h_{1}$ is a homeomorphism, we obtain a contradiction.

If $X$ is one of the examples of Slovakian spaces as constructed above, then $X \backslash D$ is connected for any countable subset $D$ of $X$. This is because we can choose a countable invariant subset $D_{0} \subset W$ with $x_{0} \in$ $D_{0}$ such that $D \subset D_{0} \times[0,1]$. Since $\operatorname{Trans}(g) \cap \operatorname{Trans}\left(g^{-1}\right)$ is residual and thus uncountable, we can choose $x \in\left(\operatorname{Trans}(g) \cap \operatorname{Trans}\left(g^{-1}\right)\right) \backslash D_{0}$. Then the orbit $\mathbb{R} x$ is connected, dense in $X$, and contained in $X \backslash D$. We don't know whether this property holds for all Slovakian spaces.

Now for our final construction.

Example 4.9. Let $C$ be a space which is countable or the Cantor set. There exists a space $K$ such that $H(K)$ is isomorphic as a topological group to the semi-direct product of $H(C)$ with $C(C, \mathbb{Z})$. The action of $H(K)$ on $K$ is topologically transitive, and every element of $H(K)$ is chain transitive. If $C$ is either finite or the Cantor set, then there exists a topologically transitive homeomorphism in $H(K)$.

We begin with $g$ a totally transitive homeomorphism on a Cantor set $W$ with a fixed point $e \in W$. Let $r:\{e\} \rightarrow\{e\}$ be the identity. The space $Y_{r}$ is the suspension of $g$ with $\{e\} \times[0,1]$ smashed to the point $e$, and $\phi_{r}$ is the associated real flow on $Y_{r}$. Then $X_{r}$ is the Slovakian space over $Y_{r}$ with $p: X_{r} \rightarrow Y_{r}$ the almost one-to-one projection. The group $H\left(X_{r}\right)$ is cyclic with generator $h_{r}$ mapped by $p$ to $\phi_{r}^{\tau}$.

Let $\left(K, e_{K}\right)$ be the smash product $\left(X_{r}, e\right) \# C$, and let $\left(L, e_{L}\right)=$ $\left(Y_{r}, e\right) \# C$. We have the projections

$$
\begin{gathered}
\pi_{L}: L \backslash\left\{e_{L}\right\}=\left(Y_{r} \backslash\{e\}\right) \times C \rightarrow C, \\
\pi=\pi_{L} \circ\left(p \times 1_{C}\right): K \backslash\left\{e_{K}\right\}=\left(X_{r} \backslash\{e\}\right) \times C \rightarrow C,
\end{gathered}
$$

and $P:\left(K, e_{K}\right) \rightarrow\left(L, e_{L}\right)$ is the smash product $(p, e) \# 1_{C}$.

If $C$ is a singleton, then $\left(K, e_{K}\right)$ is just $\left(X_{r}, e\right), H(C)$ is trivial, and $C(C, \mathbb{Z})$ is isomorphic to $\mathbb{Z}$ and to $H(K)$. The identity is chain transitive, and all other elements of $H(K)$ are topologically transitive. So the result is clear in this case. Now assume that $C$ has at least two 
points. This implies that $e_{K}$ disconnects $K$, while no other point does since no point of $X_{r}$ disconnects $X_{r}$. Hence, every homeomorphism of $K$ preserves $e_{K}$.

From the discussion preceding Example 4.3 it follows that $H(K)$ is the semi-direct product of $H(C)$ and $C\left(C, H\left(X_{r}\right)\right)$, which is essentially $C(C, \mathbb{Z})$ since $H\left(X_{r}\right)$ is Slovakian. The projection $\pi: K \backslash\left\{e_{K}\right\} \rightarrow C$ induces the group surjection $\pi_{*}: H(K) \rightarrow H(C)$, which is split by the injection $j: H(C) \rightarrow H(K)$. If $k \in H(C)$, then $j(k)$ is $\left(1_{X_{r}}, e\right) \# k$. In this case, the subgroup $C(C, \mathbb{Z})$ is commutative. If $q \in C(C, \mathbb{Z})$, then the associated element of $H(K)$ is the projection of $(x, c) \mapsto$ $\left(h_{r}^{q(c)}(x), c\right)$. The constant elements, the homeomorphisms $\left(h^{n}, e\right) \# 1_{C}$, commute with all the elements of $H(K)$ since they commute with the members of the subgroup $j(H(C))$. For $(n, k) \in \mathbb{Z} \times H(C)$ let $J(n, k)=$ $\left(h^{n}, e\right) \# k$. Thus, $J: \mathbb{Z} \times H(C) \rightarrow H(K)$ is a topological embedding and a group homomorphism.

Notice that $P$ maps $\left(h_{r}^{q(c)}(x), c\right)$ to $\left(\phi_{r}^{q(c) \tau}(x), c\right)$ and maps $(y, k(c))$ to $(p(y), k(c))$. It follows that every homeomorphism of $K$ projects by $P$ to a homeomorphism of $L$.

Case 1 ( $C$ is finite): Let $k$ be a cyclic permutation on $C$ so that $C$ consists of a single periodic orbit under $k$. Since $h$ is totally transitive, the product $h \times k$ on $X_{r} \times C$ is topologically transitive, and so it projects to a topologically transitive element of $\mathbb{Z} \times H(C) \subset H(K)$.

Let $F$ be an arbitrary homeomorphism on $K$ with $k=\pi_{*}(F)$. Since $k$ is a permutation of a finite set, there exists $N \in \mathbb{N}$ such that $k^{N}$ is the identity. This means that $F^{N}$ projects to the identity on $C$ and so preserves each fiber $X_{r} \times\{c\}$. So there exists $n_{c} \in \mathbb{Z}$ such that $F^{N}$ restricts to $h^{n_{c}}$ on the fiber. This is chain transitive on the fiber, topologically transitive if $n_{c} \neq 0$, and so every point is chain recurrent for $F^{n}$ and hence for $F$. Since $K$ is connected, $F$ is chain transitive on $K$.

Case 2 ( $C$ is countably infinite): Since $C$ is countable, the isolated points are dense in $C$. If $x_{1}, x_{2} \in C$ are distinct isolated points, let $k$ interchange these two points and fix the remaining points of $C$. By Case $1, J(1, k)=(h, e) \# k$ restricts to a topologically transitive homeomorphism on the closed subset $\left(X_{r}, e\right) \#\left\{x_{1}, x_{2}\right\}$. This implies that $H(K)$ acts in a topologically transitive manner on the set $\left(X_{r} \backslash\{e\}\right) \times \operatorname{Iso}(C)$. This is a dense open subset of $K$, and so $H(K)$ is topologically transitive on $K$.

Case 3 ( $C$ is a Cantor set): A homeomorphism $k$ on $C$ is topologically mixing if, for all nonempty open sets $U_{1}, U_{2} \subset C$, there exists $N \in \mathbb{N}$ such that $N_{k}\left(U_{1}, U_{2}\right)$ contains every $n \geq N$. The shift homeomorphism 
on $\{0,1\}^{\mathbb{Z}}$ is topologically mixing, and the product of a topologically mixing and a topologically transitive homeomorphism is topologically transitive. It follows that if $k \in H(C)$ is topologically mixing, then $J(1, k)$ is a topologically transitive element of $H(K)$.

It remains to show that every element of $H(K)$ is chain transitive. It suffices to show that, for an arbitrary $F \in H(K)$ and an arbitrary point $y$ of $K$, we have $e_{K} \in \mathcal{C} F(y)$. Applying this to $F$ and $F^{-1}$, we see that every point of $K$ is chain recurrent for $F$.

With the homeomorphism $g$ on $W$ chosen as above, we do not know whether this is always true. We prove it by imposing further restrictions on the homeomorphism $g$ with which we began.

Call a homeomorphism $g$ semi-minimal if it has a fixed point $e$ and if for every $x \neq e$ the orbit $\mathcal{O} g_{ \pm}(x)=\left\{g^{n}(x): n \in \mathbb{Z}\right\}$ is dense in $W$. Such semi-minimal homeomorphisms exist. Topologically mixing examples of semi-minimal homeomorphisms on the Cantor set are constructed explicitly in [7] Theorem 4.19, and Theorem 4.16 there shows that the semi-minimal homeomorphisms form a dense $G_{\delta}$ subset of the set of those chain transitive homeomorphisms on a Cantor set which admit a fixed point. Now assume that $g$ is a semi-minimal homeomorphism which is topologically mixing and so is totally transitive. This implies that if $x \neq e$, then the real orbit $\mathbb{R} x$ is dense in $Y_{r}$.

Let $F \in H(K)$ and $y \in K$. We must show that $y$ chains to $e_{K}$. Let $k=\pi_{*}(F)$ be a homeomorphism on $C$ and $G=P_{*}(F)$ a homeomorphism on $L$.

The invariant set $\omega F(y)$ contains a closed subset $M$ so that the restriction of $F$ to $M$ is minimal. Of course, $M \subset \mathcal{C} F(y)$. So it suffices to prove that $e_{K} \in \mathcal{C} F(M)$. This is obvious if $M=\left\{e_{K}\right\}$. Now assume $M$ is not equal to $\left\{e_{K}\right\}$ and so does not contain $e_{K}$ since distinct minimal sets are disjoint.

Hence, $M$ is a compact subset of $K \backslash\left\{e_{K}\right\}$. Since $\pi$ maps $F$ to $k$, the subset $A=\pi(M)$ of $C$ is compact and invariant on which $k$ restricts to a minimal homeomorphism. Let $\left(\widehat{K}, e_{K}\right)=\left(X_{r}, e\right) \# A$ and $\left(\widehat{L}, e_{K}\right)=\left(Y_{r}, e\right) \# A$, so that $\widehat{K}$ is a closed $F$-invariant subset of $K$ and $\widehat{L}$ is a closed $G$-invariant subset of $L$. The restriction of $F$ to $M \subset \widehat{K}$ is minimal, and so if $\widetilde{M}=P(M) \subset \widehat{L}$, then $G$ on $\widetilde{M}$ is minimal. On $\widetilde{L}$ (but not on $\widetilde{K}$ ) the homeomorphism $\left(\phi_{r}^{t}, e\right) \# 1_{A}$ is defined for every $t$, and each such homeomorphism commutes with $G$. It follows that $\widetilde{M}_{t}=\left(\phi_{r}^{t}, e\right) \# 1_{A}(\widetilde{M})$ is a $G$-invariant subset on which $G$ is minimal. If $a \in A$, there exists $((x, s), a) \in \widetilde{M}$ for some $x \in W \backslash\{e\}$ and $s \in[0,1)$ because $\pi_{L}$ maps $\widetilde{M}$ onto $A$. Because $x \neq e$, the real orbit $\mathbb{R} x$ is dense in $Y_{r}$. It follows that $\bigcup_{t} \widetilde{M}_{t}$ is dense in every fiber $\pi_{L}^{-1}(a)$. This implies 
that the union of the minimal subsets of $G$ is dense in $\widehat{L}$. Above each $\widetilde{M}_{t}$ there is a minimal subset $M_{t} \subset \widehat{K}$ with $P\left(M_{t}\right)=\widetilde{M}_{t}$. Because $P$ is an almost one-to-one map, it follows that the union $\bigcup_{t} M_{t}$ is dense in $\widehat{K}$. This implies that the recurrent points for $F$ are dense in $\widehat{K}$, and so every point of $\widehat{K}$ is chain recurrent. Since $\widehat{K}$ is connected, $F$ on $\widehat{K}$ is chain transitive and, in particular, $e_{K} \in \mathcal{C} F(M)$, as required.

Remark: Notice that an almost one-to-one lift of a chain transitive map need not be chain transitive. Let $t$ be translation by 1 on $\mathbb{Z}$ and $t^{*}, t^{* *}$ the extensions to the one-point compactification $\mathbb{Z}^{*}=\mathbb{Z} \cup\{\infty\}$ and the two-point compactification $\mathbb{Z}^{* *}=\mathbb{Z} \cup\{+\infty,-\infty\}$. The map $p: \mathbb{Z}^{* *} \rightarrow \mathbb{Z}^{*}$ which maps both $+\infty$ and $-\infty$ to $\infty$ is an almost oneto-one map, but while $t^{*}$ is chain transitive, $t^{* *}$ is not. In general, if $A$ is a nowhere dense, closed, invariant set which contains $|\mathcal{C} f|$ for a homeomorphism $f$ on $X$, then smashing $A$ to the point $e$ in $X / A$, the induced homeomorphism $f_{A}$ on $X / A$ has $e$ as the unique minimal point, so $f_{A}$ is chain transitive, the projection $q_{A}: X \rightarrow X / A$ is almost one-toone since $A$ is nowhere dense, and $f$ is not chain recurrent since $|\varrho f|$ is a proper subset of $X$. For example, with $X=I^{2}$ and $f(x, y)=\left(x^{2}, y^{2}\right)$, the corner points are the only chain recurrent points. Let $A$ be the boundary $I \times\{0,1\} \cup\{0,1\} \times I$.

\section{Appendix: Chaotic Spaces}

The rigid and strongly chaotic spaces constructed in [11] and [8] are dendrites or subsets of $\mathbb{R}^{2}$. It will be convenient for our purposes to use infinite-dimensional examples.

Let $M$ be an infinite subset of $\mathbb{N} \backslash\{1\}$, and let $m: \mathbb{N} \rightarrow M$ be the unique order preserving bijection. For $n=0,1, \ldots$, let $M^{n}=$ $\left\{m\left(2^{n}(2 k-1)\right): k \in \mathbb{N}\right\}$ so that $\left\{M^{n}\right\}$ is a partition of $M$ by a pairwise disjoint sequence of infinite sets. For all $i \in \mathbb{N}$ let $S^{i}$ be the sphere in $\mathbb{R}^{i+1}$ of radius $i^{-1}$ centered at $\left(i^{-1}, 0, \ldots, 0\right)$ so that the origin 0 is a point of $S^{i}$. Let $S(n, k)=S^{m\left(2^{n}(2 k-1)\right)}$ for $n=0,1, \ldots$ and $k \in \mathbb{N}$.

Let $Z^{0}$ be the two-torus $S^{1} \times S^{1}$, and let $A^{0}=\left\{a_{k}^{0}: k \in \mathbb{N}\right\}$ be a dense sequence of distinct points in $Z^{0}$. Let $Z^{1}$ be $Z^{0}$ with a copy of $S(0, k)$ attached to $Z^{0}$ with $0 \in S(0, k)$ identified with the attachment point $a_{k}^{0}$ for $k \in \mathbb{N}$ so that the attached spheres are disjoint in $Z^{1}$. Let $r^{1}: Z^{1} \rightarrow Z^{0}$ be the retraction with each $S(0, k)$ mapped to the point $a_{k}$. For each $k \in \mathbb{N}$ let $r(0, k): Z^{1} \rightarrow S(0, k)$ be the retraction mapping $Z^{1} \backslash S(0, k)$ to the point $a_{k}$. Since the diameters of the spheres tend to zero, the space $Z^{1}$ is compact and metrizable and the retractions are continuous. The open set $Z^{1} \backslash Z^{0}$ is dense in $Z^{1}$. 
For $n \geq 1$ assume that $Z^{n}$ has been defined with a retraction $r^{n}$ : $Z^{n} \rightarrow Z^{n-1}$ and with retractions $r(n-1, k): Z^{n} \rightarrow S(n-1, k)$ onto the attached spheres and so that $Z^{n} \backslash Z^{n-1}$ is dense in $Z^{n}$. Let $A^{n}=\left\{a_{k}^{n}\right.$ : $k \in \mathbb{N}\}$ be a sequence of distinct points dense in $Z^{n} \backslash Z^{n-1}$. Let $Z^{n+1}$ be $Z^{n}$ with a copy of $S(n, k)$ attached to $Z^{n}$ with $0 \in S(n, k)$ identified with the attachment point $a_{k}^{n}$ for $k \in \mathbb{N}$ so that the attached spheres are disjoint in $Z^{n+1}$. Let $r^{n+1}: Z^{n+1} \rightarrow Z^{n}$ be the retraction which maps the new spheres to their attachment points, and let $r(n, k): Z^{n+1} \rightarrow$ $S(n, k)$ be the retraction mapping $Z^{n+1} \backslash S(n, k)$ to $a_{k}^{n}$. Notice that for each $x \in Z^{n} \backslash A^{n}$ the set $\left(r^{n+1}\right)^{-1}(x)=\{x\}$.

Let $Z$ be the inverse limit of the system $\left\{r^{n}: Z^{n} \rightarrow Z^{n-1}, n \in\right.$ $\mathbb{N}\}$. The inclusions $i_{n}: Z^{n} \rightarrow Z^{m}$ with $m>n$ commute with the retractions. We obtain a limiting inclusion and so can regard $\left\{Z^{n}\right\}$ as an increasing sequence of subsets of $Z$. The projection $r_{n}: Z \rightarrow Z^{n}$ is then a retraction with $r^{n} \circ r_{n}=r_{n-1}$. We write $r(n, k): Z \rightarrow S(n, k)$ for the composition of retractions $r(n, k) \circ r_{n+1}$. Notice that if we pick $z$ from the set $Z^{*}=Z \backslash\left(\bigcup_{n} Z^{n}\right)$, then there is a unique sequence of attachment points $\left\{a_{k_{n}}^{n}\right\}$ converging to $z$ with $r^{n}\left(a_{k_{n}}^{n}\right)=a_{k_{n-1}}^{n-1}$ for all $n$. Thus, $Z^{*}$, while a dense $G_{\delta}$, is totally disconnected.

Observe that $\left(r_{n+1}\right)^{-1}(S(n, k))$ and $\left\{a_{k}^{n}\right\} \cup\left(r_{n}\right)^{-1}\left(Z^{n} \backslash\left\{a_{k}^{n}\right\}\right)$ are connected sets with union $Z$ and which meet only at $a_{k}^{n}$. Thus, each attachment point disconnects $Z$.

If $F \subset Z$ is a finite set containing no attachment points, then $Z \backslash F$ is connected. For any finite $F \subset Z$, the set $Z \backslash F$ contains only finitely many components. In fact, the number of components is exactly one more than the number of attachment points in $F$.

Lemma 5.1. If $W$ is a closed, connected, nontrivial subset of $Z \times I^{N}$ such that $W \backslash A$ is connected for any $A \subset W$ with topological dimension at most $N$, then either $W \subset Z^{0} \times I^{N}$ or there exists a unique attached sphere $S(n, k)$ such that $W \subset S(n, k) \times I^{N}$.

Proof: Notice first that the dimension of $W$ is at least $N+2$. For if $U$ is a nonempty open set with $\bar{U}$ a proper subset of $W$, then the topological boundary of $U$ disconnects $W$ and so has dimension at least $N+1$. This implies that the dimension of $W$ is at least $N+2$.

The set $W$ is not contained in $Z^{*} \times I^{N}$ since $Z^{*}$ is totally disconnected and the components of $Z^{*} \times I^{N}$ have dimension $N$. Assume $W$ is not a subset of $Z^{0} \times I^{N}$.

If $W$ meets $\left(Z^{n+1} \backslash Z^{n}\right) \times I^{N}$ for some $n=0,1, \ldots$, then $X$ meets $\left(S(n, k) \backslash\left\{a_{k}^{n}\right\}\right) \times I^{N}$ for some $k$. Since $a_{k}^{n} \times I^{N}$ disconnects $Z \times I^{N}$ and no such set disconnects $W$, it follows that $W \subset r_{n}^{-1}(S(n, k)) \times I^{N}$. For the attachment points $a_{j}^{n+1}$ in $S(n, k) \backslash\left\{a_{k}^{n}\right\}$, the sets $a_{j}^{n+1} \times I^{N}$ 
also disconnect $Z$. We see that either $W \subset S(n, k) \times I^{N}$ or else $W \subset$ $r_{n+1}^{-1}(b) \times I^{N}$, where $b$ is one of the attachment points in $S(n, k) \backslash\left\{a_{k}^{n}\right\}$.

If this process does not halt with $W$ contained in the product of $I^{N}$ with some attached sphere, then there is a sequence of attachment points $\left\{b_{k_{i}}^{n_{i}}\right\}$ with $W \subset r_{n_{i}+1}^{-1}\left(b_{k_{i}}^{n_{i}}\right) \times I^{N}$ and $r_{n_{i}+1}\left(b_{k_{i+1}}^{n_{i+1}}\right)=b_{k_{i}}^{n_{i}}$ with $n_{i} \longrightarrow \infty$. The limit of such a sequence $\left\{b_{k_{i}}^{n_{i}}\right\}$ is a point of $Z^{*}$, and this would imply that $W$ is a subset of $Z^{*} \times I^{N}$.

If $A$ and $X$ are spaces, a non-null embedding of $A$ in $X$ is a continuous injective function $j: A \rightarrow X$ which is not homotopic to a constant map in $X$.

Corollary 5.2. If $S$ is a sphere of dimension at least two and $j$ : $S \times I^{N} \rightarrow Z \times I^{N}$ is a non-null embedding, then for a unique pair $(n, k)$, we have $j\left(S \times I^{N}\right) \subset S(n, k) \times I^{N}$. Furthermore, the dimension of $S$ equals the dimension of $S(n, k)$. On the other hand, if $j_{0}: S \rightarrow S(n, k)$ is a homeomorphism, then $j=j_{0} \times 1_{I^{N}}: S \times I^{N} \rightarrow Z \times I^{N}$ is a non-null embedding.

Proof: Corollary 1 of Theorem IV.4 in [15] says that a connected manifold of dimension at least $N+2$ cannot be disconnected by a subset of dimension $N$. Hence, Lemma 5.1 applies to $W=j\left(S \times I^{N}\right)$, and so $j\left(S \times I^{N}\right) \subset S(n, k) \times I^{N}$ for some pair $(n, k)$ which is clearly unique. The space $S \times I^{N}$ cannot be embedded in a manifold of smaller dimension, and so $\operatorname{dim} S \leq \operatorname{dim} S(n, k)$. If the inequality were strict then the map $j$ would be homotopically trivial since the homotopy groups of a sphere vanish below its dimension. Hence, the dimension of $S$ must be $m\left(2^{n}(2 k-1)\right)=\operatorname{dim} S(n, k)$.

If $j_{0}: S \rightarrow S(n, k)$ is a homeomorphism, then $j=j_{0} \times 1_{I^{N}}: S \times I^{N} \rightarrow$ $S(n, k) \times I^{N}$ is not homotopically trivial. Since $S(n, k)$ is a retract of $Z$, the embedding $j: S \times I^{N} \rightarrow Z \times I^{N}$ is not homotopically trivial.

Thus, we can associate to any open subset $U \subset Z$ the set $\delta(U)=$ $\{\operatorname{dim} S(n, k): S(n, k) \subset U\} \subset M$. Since the diameters of the attaching spheres tend to zero, it follows that if $U$ is nonempty, then $\delta(U)$ is infinite.

Corollary 5.2 says that for a sphere $S$ of dimension at least two there exists a non-null embedding of $S \times I^{N}$ into $U \times I^{N}$ iff $\operatorname{dim} S \in \delta(U)$. That is, $\delta(U)$ is a topological invariant for the sets $U \times I^{N}$. Observe that if $U_{1}$ and $U_{2}$ are disjoint, nonempty, open sets in $Z$, then $\delta\left(U_{1}\right) \cap \delta\left(U_{2}\right)=$ $\emptyset$ since distinct attached spaces have distinct dimensions.

If we begin by partitioning $\mathbb{N} \backslash\{1\}$ into a pairwise disjoint sequence $\left\{M_{n}\right\}$ of infinite subsets, then we can do this construction associating 
CHAIN TRANSITIVE HOMEOMORPHISMS ON A SPACE: ALL OR NONE 49

$Z_{n}$ with $M_{n}$. That is, $\delta\left(Z_{n}\right)=M_{n}$. It follows that if $U_{1} \subset Z_{n_{1}}, U_{2} \subset Z_{n_{2}}$ are nonempty open subsets with $n_{1} \neq n_{2}$, then $\delta\left(U_{1}\right) \cap \delta\left(U_{2}\right)=\emptyset$.

Condition $(*)$ of Section is thus proved for this sequence of spaces.

\section{References}

1. S. Agronsky, A. Bruckner, J. Ceder, T. Pearson (1989-90) The structure of $\omega$-limit sets for continuous functions, Real Analysis Exchange 15:483510.

2. S. Agronsky and J. Ceder (1991-92) What sets can be $\omega$-limit sets in $E^{n}$ ?, Real Analysis Exchange 17:97109.

3. S. Agronsky and J. Ceder (1991-92) Each Peano subspace of $E^{k}$ is an $\omega$-limit set, Real Analysis Exchange 17:371378.

4. E. Akin (1993) The general topology of dynamical systems, Amer. Math. Soc., Providence, RI.

5. E. Akin (2004) Lectures on Cantor and Mycielski sets for dynamical systems, Contemp. Math. 346:23-79

6. E. Akin, E. and J. Carleson (2012) Conceptions of Topological Transitivity, Topology and its Applications, 159:2815-2830.

7. E. Akin (2015) Conjugacy in the Cantor set automorphism group (to appear Contemp. Math.).

8. J. J. Charatonik and W. J. Charatonik (1996) Strongly chaotic dendrites, Colloq. Math. 70:181-190.

9. C. Conley (1978) Isolated invariant sets and the Morse index, CBMS Regional Conf. Series in Math., vol. 38, Amer. Math. Soc., Providence, RI.

10. J. de Groot (1959) Groups represented by homeomorphism groups, I Math. Ann. 138:80-102.

11. J. de Groot and R. J. Willie (1958) Rigid continua and topological group-pictures Archiv. der Math. 9:441-446.

12. Y. N. Dowker and F. G. Friedlander (1954) On limit sets in dynamical systems, Proc. London Math. Soc. (3) 4:168176.

13. T. Downarowicz, L. Snoha, D. Tywoniuk (2015)- Minimal spaces with cyclic group of homeomorphisms (to appear) ArXiv: 1503.03246.

14. A. Gutek (1979) On extending homeomorphisms on the Cantor set, Topological Structures II, Mathematical Centre Tracts 115:105-116.

15. W. Hurewicz and H. Wallman (1941) Dimension theory, Princeton U. Press, Princeton, NJ.

16. J. Kelley (1955) General topology, Van Nostrand, Princeton, NJ.

17. B. Knaster and M. Reichbach (1953) Notion d'homogeneite et prologements de homeomorphies, Fund. Math. 40:180-193.

18. E. R. Lorch (1981) On some properties of the metric subalgebras of $\ell^{\infty}$, Integral Equations and Operator Theory, 4/3: 422-434.

19. E. R. Lorch (1985) Certain compact spaces and their homeomorphism groups, Rend. Seminario Mat. e Fis., Milano, 52: 75-86. 
20. A. N. Sarkovskii (1965) Attracting and attracted sets, Dokl. Akad. Nauk SSSR 160:10361038. English translation in Sov. Math. Dokl. (1965), 6:268270.

21. A. G. Sivak (2003) On the structure of transitive $\omega$-limit sets for continuous maps, Qual. Theory of Dyn. Sys. 4:109123.

22. T. Tsankov (2006) Compactifications of $\mathbb{N}$ and Polishable subgroups of $S_{\infty}$, Fund. Math. 189:269-284.

Mathematics Department, The City College, 137 Street and Convent Avenue, New York City, NY 10031, USA

E-mail address: ethanakin@earthlink.net

University of Oulu, Department of Mathematical Sciences, PB 8000, FI-90014 OUlun Yliopisto, Finland.

E-mail address: Juho.Rautio@oulu.fi 